\title{
An Application of Homotopy Perturbation Method to Fractional-Order Thin Film Flow of the Johnson-Segalman Fluid Model
}

\author{
Mubashir Qayyum (iD, ${ }^{1}$ Farnaz Ismail, ${ }^{2}$ Syed Inayat Ali Shah, ${ }^{2}$ Muhammad Sohail $\mathbb{D},{ }^{3}$ \\ Essam R. El-Zahar, ${ }^{4,5}$ and K. C Gokul $\mathbb{1}^{6}$ \\ ${ }^{1}$ Department of Sciences and Humanities, National University of Computer \& Emerging Sciences FAST Lahore, Lahore, Pakistan \\ ${ }^{2}$ Department of Mathematics, Islamia College University Peshawar, Peshawar, Pakistan \\ ${ }^{3}$ Department of Applied Mathematics and Statistics, Institute of Space Technology, P.O. Box 2750, Islamabad, Pakistan \\ ${ }^{4}$ Department of Mathematics, College of Science and Humanities in Al-Kharj, Prince Sattam Bin Abdulaziz University, \\ P.O. Box 83, Al-Kharj 11942, Saudi Arabia \\ ${ }^{5}$ Department of Basic Engineering Science, Faculty of Engineering, Menoufia University, Shebin El-Kom 32511, Egypt \\ ${ }^{6}$ Department of Natural Sciences (Mathematics Group), Kathmandu University, Kathmandu, Nepal
}

Correspondence should be addressed to K. C Gokul; gokul.kc@ku.edu.np

Received 31 December 2021; Revised 22 January 2022; Accepted 31 January 2022; Published 23 February 2022

Academic Editor: Arshad Riaz

Copyright (c) 2022 Mubashir Qayyum et al. This is an open access article distributed under the Creative Commons Attribution License, which permits unrestricted use, distribution, and reproduction in any medium, provided the original work is properly cited.

\begin{abstract}
Thin film flow is an important theme in fluid mechanics and has many industrial applications. These flows can be observed in oil refinement process, laser cutting, and nuclear reactors. In this theoretical study, we explore thin film flow of non-Newtonian Johnson-Segalman fluid on a vertical belt in fractional space in lifting and drainage scenarios. Modelled fractional-order boundary value problems are solved numerically using the homotopy perturbation method along with Caputo definition of fractional derivative. In this study, instantaneous and average velocities and volumetric flux are computed in lifting and drainage cases. Validity and convergence of homotopy-based solutions are confirmed by finding residual errors in each case. Moreover, the consequences of different fractional and fluid parameters are graphically studied on the velocity profile. Analysis shows that fractional parameters have opposite effects of the fluid velocity.
\end{abstract}

\section{Introduction}

Modelling and analysis of non-Newtonian fluids is an important and active research theme in industrial engineering. Food processing, paper production, blood flow analysis, and mud drilling are different applications areas of non-Newtonian fluids. These fluids are defined through a nonlinear relationship between rate of deformation and stress tensors, and therefore, it has several models in different scenarios. Johnson-Segalman fluid is one of the very significant fluid modals which have numerous engineering and industrial applications.

Thin film flow can be detected in different natural situations, for example, movement of raindrop on window glass, tears in the eyes, and lava flow. Industrial applications of such flows include oil refining, nuclear reactors, and laser cutting[1-4]. The initial work on thin film flow was carried out in [3] for Newtonian fluids, but this study has limitations and cannot be generalized for non-Newtonian fluids such as melted plastics, gels, pastes, honey, ketchup, and blood [5]. Siddiqui et al. examined thin film of different fluids including PTT (Phan-Thien and Tanner) and third and fourth grade fluids in [6-8]. Landau [9] and Stuart [10] extended these analyses to turbulence. Ullah et al. studied the film flow under slip conditions in generalized Maxwell fluids in [11]. Ruan et al. studied thin film from a distributed source on a vertical wall in [12]. Ahmad and Xu proposed an improved nanofluid model in thin film under the action of gravity in [13]. 
Fractional calculus has gained significant attention due to its striking application as a new modelling tool in a variety of fields, such as fluid dynamics, hydrology, system control theory, signal processing, physics, biology, and finance [14-20]. These fractional models are more appropriate for describing memory and transmissible properties of different materials than integral-order models. In the past few decades, various numerical techniques have been developed by different researchers for nonlinear BVPs. Wazwaz proposed the modified decomposition method (MDM) for BVPs in [21]. Noor and Mohyud-Din used VIM along with He's polynomials for the solution of higher order BVPs [22]. Liu et al. used the multiscale method for nanoparticle diffusion in sheared cellular blood flow in [23]. Jafari and Gejji used adomian decomposition for the solution of system of FDEs in [24]. Hashim et al. apply OHAM to fractional-order fuzzy differential equations in [22]. Rysak and Gregorczyk proposed DTM for fractional dynamical systems in [25]. Zada et al. apply NIM to fractional PDEs in [26]. Yaghouti used radial basis functions to different families of fractional differential equations in [27]. Al-Kuze et al. used spectral quasi-linearization and irreversibility analysis of magnetized cross-fluid flow [28].

In this study, we extend the theoretical study of thin film flow to fractional space in case of non-Newtonian Johnson-Segalman fluid since exact solutions of highly nonlinear fractional differential equations (FDEs) are not possible. The conventional approach is to use perturbation techniques in such scenarios, which again need small or large parameters. To avoid this, we utilize the well-known homotopy perturbation method (HPM) [29-33] along with fractional calculus for solution purpose. This method is effectively used by many scholars in [29-33]. Validation and convergence of the obtained numerical solutions are confirmed by means of finding residual errors. To the best of the authors' knowledge, the given problem has not been attempted before in fractional space.

\section{Preliminaries}

2.1. Fractional Calculus. Few basic definitions of fractional calculus are given below.

Definition 1. Let $f(t), t>0$, be a real function in the space $C_{\mu}, \mu \in R$, if $\exists$ a real number $p>\mu \ni f(t)=t^{p} f_{1}$ $(t)$, where $f_{1}(t) \in C(0, \infty)$, and it is in the space

$$
C_{\mu}^{m} \Leftrightarrow f^{m} \in C_{\mu}, m \in N .
$$

Definition 2. The Caputo fractional derivative $D^{\alpha}$ is defined as

$$
\begin{aligned}
D^{\alpha} f(t)= & \frac{1}{\Gamma(m-\alpha)} \int_{0}^{t}(t-\tau)^{m-\alpha-1} f^{m} d \tau, \text { for } m \\
& -1<\alpha<m, m \in N, t>0 .
\end{aligned}
$$

Definition 3. $\alpha \geq 0$ order Riemann-Liouville fractional integration is defined as
$J^{\alpha} f(t)=\frac{1}{\Gamma(\alpha)} \int_{0}^{t}(t-\tau)^{\alpha-1} f(\tau) d \tau, k-1<\alpha<k$, and $k \in N$.

2.2. Basic Equations. The basic equations of incompressible fluid of Johnson-Segalman fluid are

$$
\begin{aligned}
& \operatorname{div} \mathscr{V}=0, \\
& \rho \frac{\mathrm{D} \mathscr{V}}{\mathrm{D} t}=\operatorname{div} \boldsymbol{\sigma}+\rho \mathbf{f},
\end{aligned}
$$

where $\mathscr{V}$ is the velocity vector, $\rho$ is the constant density, $\mathbf{f}$ is the body force per unit mass, and $\sigma$ is the Cauchy stress tensor, where

$$
\begin{gathered}
\boldsymbol{\sigma}=-p \mathbf{I}+\mathbf{T}, \\
\mathbf{T}=\mathbf{S}+2 \mu \mathbf{D}, \\
\mathbf{S}+m\left[(\mathbf{W}-a \mathbf{D})^{T} \mathbf{S}+\mathbf{S}(\mathbf{W}-a \mathbf{D})+\frac{\mathrm{D} \mathbf{S}}{\mathrm{D} t}\right]=2 \mathbf{D} \eta .
\end{gathered}
$$

\section{Mathematical Formulation in Lifting Case}

The belt is passing through the container filled with Johnson-Segalman fluid, and it is moving vertically with constant speed $U_{0}$. A uniform thickness $\delta$ of the thin film is taken up by the belt, but due to gravity fluid, it is draining down. Let pressure be assumed to be atmospheric, and the flow is uniform, steady, and laminar. Also, the $x$-axis is considered to be normal, while the $y$-axis is along the belt.

The boundary conditions are

$$
\begin{aligned}
x & =0, \\
v & =U_{0}, \\
x & =\delta, \\
T_{x y} & =0 .
\end{aligned}
$$

Velocity is of the form

$$
\begin{gathered}
\mathscr{V}=(0, v(x), 0), \\
\mathbf{T}=\mathbf{T}(x) \text { and } \mathbf{T}_{\mathbf{x z}},
\end{gathered}
$$

where the extra and shear stress tensor is $\mathbf{T}=\mathbf{T}(x)$ and $\mathbf{T}_{\mathbf{x z}}$, respectively.

Substitution of (8) in (3) and (4) shows that (3) is satisfied, while (4) has the form

$$
\begin{aligned}
& 0=\frac{\mathrm{d} T_{\mathrm{xx}}}{\mathrm{d} x}+\rho f_{1}, \\
& 0=\frac{\mathrm{d} T_{\mathrm{xy}}}{\mathrm{d} x}+\rho f_{2},
\end{aligned}
$$

where $f_{1}$ and $f_{2}$ are the components of body force. 
Since gravitational force is in downward while $y$-axis is in upward directions, hence, (12) and (13) become

$$
\begin{aligned}
& 0=\frac{d T_{x x}}{d x}, \\
& 0=\frac{d T_{x y}}{d x}-\rho g .
\end{aligned}
$$

By using equations (8), (10), and (11) in (7), components of $\mathbf{S}$ are

$$
\begin{aligned}
& S_{\mathrm{xx}}=\frac{-\eta m(1-\mathrm{a})(\mathrm{d} v / \mathrm{d} x)^{2}}{1+m^{2}\left(1-a^{2}\right)(\mathrm{d} v / \mathrm{d} x)^{2}}, \\
& S_{x y}=\frac{\eta \mathrm{d} v / \mathrm{d} x}{1+m^{2}\left(1-a^{2}\right)(\mathrm{d} v / \mathrm{d} x)^{2}}=S_{y x}, \\
& S_{\mathrm{yy}}=\frac{\eta m(1+\mathrm{a})(\mathrm{d} v / \mathrm{d} x)^{2}}{1+m^{2}\left(1-a^{2}\right)(\mathrm{d} v / \mathrm{d} x)^{2}} .
\end{aligned}
$$

Using (15)-(17), stress tensor $\mathbf{T}$ in (6) is

$$
\begin{aligned}
& T_{\mathrm{xx}}=\frac{-\eta m(1-\mathrm{a})(\mathrm{d} v / \mathrm{d} x)^{2}}{1+m^{2}\left(1-a^{2}\right)(\mathrm{d} v / \mathrm{d} x)^{2}}, \\
& T_{\mathrm{xy}}=\mu\left(\frac{\mathrm{d} v}{\mathrm{~d} x}\right)+\frac{\eta \mathrm{d} v / \mathrm{d} x}{1+m^{2}\left(1-a^{2}\right)(\mathrm{d} v / \mathrm{d} x)^{2}}=T_{\mathrm{yx}}, \\
& T_{\mathrm{yy}}=\frac{\eta m(1+\mathrm{a})(\mathrm{d} v / \mathrm{d} x)^{2}}{1+m^{2}\left(1-a^{2}\right)(\mathrm{d} v / \mathrm{d} x)^{2}} .
\end{aligned}
$$

Substituting $T_{\mathrm{xy}}$ in (14) gives

$$
\frac{d}{\mathrm{~d} x}\left[\mu\left(\frac{\mathrm{d} v}{\mathrm{~d} x}\right)+\frac{\eta \mathrm{d} v / \mathrm{d} x}{1+m^{2}\left(1-a^{2}\right)(\mathrm{d} v / \mathrm{d} x)^{2}}\right]=\rho g .
$$

Boundary conditions become

$$
\begin{gathered}
\frac{\mathrm{d} v}{\mathrm{~d} x}=0 \text { at } x=\delta, \\
v=U_{0} \text { at } x=0 .
\end{gathered}
$$

Introduce nondimensional parameters as

$$
x^{*}=\frac{x}{\delta}, v^{*}=\frac{v}{U_{0}} \text { and } \varphi=\frac{\mu}{\mu+\eta},
$$

where $\varphi$ is the ratios of viscosities. Equations (21)-(23) become

$$
\frac{d}{\mathrm{~d} x}\left[\varphi\left(\frac{\mathrm{d} v}{\mathrm{~d} x}\right)+\frac{(1-\varphi) \mathrm{d} v / \mathrm{d} x}{1+W_{e}^{2}\left(1-a^{2}\right)(\mathrm{d} v / \mathrm{d} x)^{2}}\right]=S_{t}
$$

$$
\begin{aligned}
& \text { At } x=1, \frac{\mathrm{d} v}{\mathrm{~d} x}=0 . \\
& \text { At } x=0, \quad v=1,
\end{aligned}
$$

where $S_{t}=\rho g \delta^{2} / \mu_{\text {eff }} U_{0}$ and $W_{e}=m U_{0} / \delta$ are Stokes and Weissenberg numbers, respectively, and $\mu_{\text {eff }}=\mu+\eta$.

Simplification of equation (25) gives

$$
\begin{array}{r}
\frac{d^{2} v}{\mathrm{~d} x^{2}}+W_{e}^{4}\left(1-a^{2}\right)^{2}[ \\
\frac{\mathrm{d} v}{\mathrm{~d} x}=0 \mathrm{atx}=1, \\
v=1 \text { atx }=0 .
\end{array}
$$

Now, using definition of fractional calculus given in Section 2.1, the fractional form of equation (28) is

$$
v(0)=1, v^{\prime}(1)=0,0<\alpha<1 \text {. }
$$

$$
\begin{aligned}
& \frac{d^{2} v(x)}{\mathrm{d} x^{2}}+W_{e}^{4}\left(1-a^{2}\right)^{2}\left[\varphi \frac{d^{2} v(x)}{\mathrm{d} x^{2}}-S_{t}\right]\left(D^{\alpha} v(x)\right)^{4} \\
& \quad-W_{e}^{2}\left(1-a^{2}\right)\left[\frac{d^{2} v(x)}{\mathrm{d} x^{2}}+\varphi \frac{d^{2} v(x)}{\mathrm{d} x^{2}}+2 S_{t}\right]\left(D^{\alpha} v(x)\right)^{2}-S_{t}=0,
\end{aligned}
$$

with 


\section{Homotopy Solution of Johnson-Segalman \\ Fluid in the Lifting Case}

Homotopy $\Omega \times[0,1] \longrightarrow \Re$ for (30) is

$$
\begin{aligned}
& (1-p) \frac{d^{2} v(x)}{\mathrm{d} x^{2}}+p\left[\frac{d^{2} v(x)}{\mathrm{d} x^{2}}+W_{e}^{4}\left(1-a^{2}\right)^{2}\left[\varphi \frac{d^{2} v(x)}{\mathrm{d} x^{2}}-S_{t}\right]\left(D^{\alpha} v(x)\right)^{4}\right. \\
& \left.-W_{e}^{2}\left(1-a^{2}\right)\left[\frac{d^{2} v(x)}{\mathrm{d} x^{2}}+\varphi \frac{d^{2} v(x)}{\mathrm{d} x^{2}}+2 S_{t}\right]\left(D^{\alpha} v(x)\right)^{2}-S_{t}\right]=0 . \\
& v_{0}^{\prime \prime}(\mathrm{x})=0, \quad v_{0}(0)=1, v_{0}^{\prime}(1)=0 .
\end{aligned}
$$

Using (30) and (31), we obtain the following.

Zeroth-order problem:

First-order problem:

$$
\begin{aligned}
& -S_{t}-2 S_{t} W_{e}^{2}\left(D^{\alpha} v_{0}(x)\right)^{2}+2 a^{2} S_{t} W_{e}^{2}\left(D^{\alpha} v_{0}(x)\right)^{2}-S_{t} W_{e}^{4}\left(D^{\alpha} v_{0}(x)\right)^{4} \\
& \quad+2 a^{2} S_{t} W_{e}^{4}\left(D^{\alpha} v_{0}(x)\right)^{4}-a^{4} S_{t} W_{e}^{4}\left(D^{\alpha} v_{0}(x)\right)^{4}-W_{e}^{2}\left(D^{\alpha} v_{0}(x)\right)^{2} v_{0}^{\prime \prime}(x) \\
& \quad+a^{2} W_{e}\left(D^{\alpha} v_{0}(x)\right)^{2} v_{0}^{\prime \prime}(\mathrm{x})-W_{e}^{2} \phi\left(D^{\alpha} v_{0}(\mathrm{x})\right)^{2} v_{0}^{\prime \prime}(x)+a^{2} W_{e}^{2} \phi\left(D^{\alpha} v_{0}(x)\right)^{2} v_{0}^{\prime \prime}(x) \\
& \quad+W_{e}^{4} \phi\left(D^{\alpha} v_{0}(x)\right)^{4} v_{0}^{\prime \prime}(x)-2 a^{2} W_{e}^{4} \phi\left(D^{\alpha} v_{0}(x)\right)^{4} v_{0}^{\prime \prime}(x)+a^{4} W_{e}^{4} \phi\left(D^{\alpha} v_{0}(x)\right)^{4} v_{0}^{\prime \prime}(x) \\
& \quad+v_{1}^{\prime \prime}(x)=0, v_{1}(0)=0, v_{1}^{\prime}(1)=0 .
\end{aligned}
$$

Second-order problem:

$$
\begin{aligned}
& W_{e}^{2}\left(D^{\alpha} v_{0}(x)\right)\left(D^{\alpha} v_{1}(x)\right)+4 a^{2} S_{t} W_{e}^{2}\left(D^{\alpha} v_{0}(x)\right)-4 S_{t}\left(D^{\alpha} v_{1}(x)\right) \\
& \quad-4 S_{t} W_{e}^{4}\left(D^{\alpha} v_{0}(x)\right)^{3}\left(D^{\alpha} v_{1}(x)\right) 8 a^{2} S_{t} W_{e}^{4}\left(D^{\alpha} v_{0}(x)\right)^{3}\left(D^{\alpha} v_{1}(x)\right) \\
& \quad-4 a^{4} S_{t} W_{e}^{4}\left(D^{\alpha} v_{0}(x)\right)^{3}\left(D^{\alpha} v_{1}(x)\right)-2 W_{e}^{2}\left(D^{\alpha} v_{0}(x)\right)\left(D^{\alpha} v_{1}(x)\right) v_{0}^{\prime \prime}(x) \\
& +2 a^{2} W_{e}^{2}\left(D^{\alpha} v_{0}(x)\right)\left(D^{\alpha} v_{1}(x)\right) v_{0}^{\prime \prime}(\mathrm{x})-2 W_{e}^{2} \phi\left(D^{\alpha} v_{0}(x)\right)\left(D^{\alpha} v_{1}(x)\right) v_{0}^{\prime \prime}(\mathrm{x}) \\
& +2 a^{2} W_{e}^{2} \phi\left(D^{\alpha} v_{0}(x)\right)\left(D^{\alpha} v_{1}(x)\right) v_{0}^{\prime \prime}(x)+4 W_{e}^{4} \phi\left(D^{\alpha} v_{0}(x)\right)^{3}\left(D^{\alpha} v_{1}(x)\right) v_{0}^{\prime \prime}(x) \\
& \quad-8 a^{2} W_{e}^{4} \phi\left(D^{\alpha} v_{0}(x)\right)^{3}\left(D^{\alpha} v_{1}(x)\right) v_{0}^{\prime \prime}(x)+4 a^{4} W_{e}^{4} \phi\left(D^{\alpha} v_{0}(x)\right)^{3}\left(D^{\alpha} v_{1}(x)\right) v_{0}^{\prime \prime}(x) \\
& \quad-W_{e}^{2}\left(D^{\alpha} v_{0}(x)\right)^{2} v_{1}^{\prime \prime}(x)+a^{2} W_{e}^{2}\left(D^{\alpha} v_{0}(x)\right)^{2} v_{1}^{\prime \prime}(x)-W_{e}^{2} \phi\left(D^{\alpha} v_{0}(x)\right)^{2} v_{1}^{\prime \prime}(x) \\
& +a^{2} W_{e}^{2} \phi\left(D^{\alpha} v_{0}(x)\right)^{2} v_{1}^{\prime \prime}(\mathrm{x})+W_{e}^{4} \phi\left(D^{\alpha} v_{0}(x)\right)^{4} v_{1}^{\prime \prime}(\mathrm{x})-2 a^{2} W_{e}^{4} \phi\left(D^{\alpha} v_{0}(x)\right)^{4} v_{1}^{\prime \prime}(x) \\
& +a^{4} W_{e}^{4} \phi\left(D^{\alpha} v_{0}(x)\right)^{4} v_{1}^{\prime \prime}(x)+v_{2}^{\prime \prime}(x)=0, v_{2}(0)=0, v_{2}^{\prime}(1)=0 .
\end{aligned}
$$

Similarly, we can find higher order problems and their solutions.
Third-order approximate solution, after applying Caputo definition and keeping $\alpha=0.99, \phi=0.1, S_{t}=0.01$, $a=0.01$, and $W_{e}=0.1$ fixed, is

$$
\begin{aligned}
V(x)= & 1+\frac{1}{2}\left(-0.02 t+0.01 x^{2}\right)+\left(0 . 0 7 9 6 7 1 1 \left(-8.24342 \times 10^{-8} x^{2.98}\right.\right. \\
& \frac{\left.\left.+1.23219 \times 10^{-7} \mathrm{x}^{4}-8.24098 \times 10^{-8} \mathrm{x}^{5}+2.04999 \times 10^{-8} \mathrm{x}^{6}\right)\right)}{x^{1.98}}
\end{aligned}
$$




$$
\begin{aligned}
& +\left(0 . 0 3 9 8 3 5 5 \left(-9.06776 \times 10^{-8} x^{2.98}+1.35541 \times 10^{-7} x^{4}\right.\right. \\
& \frac{\left.\left.-9.06508 \times 10^{-8} x^{5}+2.25499 \times 10^{-8} x^{6}\right)\right)}{x^{1.98}}
\end{aligned}
$$

The residual is denoted by $R$ and is defined as

$$
\begin{aligned}
R= & \frac{d^{2} V(x)}{\mathrm{d} x^{2}}+W_{e}^{4}\left(1-a^{2}\right)^{2}\left[\varphi \frac{d^{2} V(x)}{\mathrm{d} x^{2}}-S_{t}\right]\left(D^{\alpha} V(x)\right)^{4} \\
& -W_{e}^{2}\left(1-a^{2}\right)\left[\frac{d^{2} V(x)}{\mathrm{d} x^{2}}+\varphi \frac{d^{2} V(x)}{\mathrm{d} x^{2}}+2 S_{t}\right]\left(D^{\alpha} V(x)\right)^{2}-S_{t} .
\end{aligned}
$$

\section{Flow Rate and Average Velocity in the Lifting Case}

Flow rate in this case is

$$
\begin{aligned}
Q= & \frac{1}{6\left(15-16 \alpha+4 \alpha^{2}\right) \Gamma(4-\alpha)^{2}}(-3+2 \alpha)\left(-\frac{1}{-7+2 \alpha}\left(3\left(-1+a^{2}\right) S_{t}^{3} W_{e}^{2}(-3+\alpha)^{2}(-16+\alpha(25+\alpha(-13+2 \alpha)))(3+\phi)\right)\right. \\
& \left.-2\left(-3+S_{t}\right)(-5+2 \alpha) \Gamma(4-\alpha)^{2}\right),
\end{aligned}
$$

and average velocity is

$$
\begin{aligned}
\bar{V}= & \frac{1}{6\left(15-16 \alpha+4 \alpha^{2}\right) \Gamma(4-\alpha)^{2}}(-3+2 \alpha)\left(-\frac{1}{-7+2 \alpha}\left(3\left(-1+a^{2}\right) S_{t}^{3} W_{e}^{2}(-3+\alpha)^{2}(-16+\alpha(25+\alpha(-13+2 \alpha)))(3+\phi)\right)\right. \\
& \left.-2\left(-3+S_{t}\right)(-5+2 \alpha) \Gamma(4-\alpha)^{2}\right) .
\end{aligned}
$$

\section{Mathematical Formulation in the \\ Drainage Case}

Let fluid be draining down due to gravity on the infinite stationary belt. Equations (4)-(8) become

$$
0=\frac{\mathrm{d} T_{\mathrm{xy}}}{\mathrm{d} x}+\rho g \text {. }
$$

Using equation (19) in (14) gives the form

$$
\frac{d}{\mathrm{~d} x}\left[\mu\left(\frac{\mathrm{d} v}{\mathrm{~d} x}\right)+\frac{\eta \mathrm{d} v / \mathrm{d} x}{1+m^{2}\left(1-a^{2}\right)(\mathrm{d} v / \mathrm{d} x)^{2}}\right]=-\rho g
$$

with

$$
\begin{gathered}
\frac{\mathrm{d} v}{\mathrm{~d} x}=0 \text { at } x=\delta, \\
v=0 \text { at } x=0 .
\end{gathered}
$$

Equation (40) in the dimensionless form is

$$
\frac{d}{\mathrm{~d} x}\left[\frac{(1-\varphi) \mathrm{d} v / \mathrm{d} x}{1+W_{e}^{2}\left(1-a^{2}\right)(\mathrm{d} v / \mathrm{d} x)^{2}}+\varphi\left(\frac{\mathrm{d} v}{\mathrm{~d} x}\right)\right]=-S_{t} .
$$

$$
\begin{aligned}
& \text { At } x=1, \frac{\mathrm{d} v}{\mathrm{~d} x}=0, \\
& \text { At } x=0, \quad v=0 .
\end{aligned}
$$

Simplification of (43) gives

$$
\begin{aligned}
& \frac{d^{2} v}{\mathrm{~d} x^{2}}+W_{e}^{4}\left(1-a^{2}\right)^{2}\left[\varphi \frac{d^{2} v}{\mathrm{~d} x^{2}}+S_{t}\right]\left(\frac{\mathrm{d} v}{\mathrm{~d} x}\right)^{4} \\
& \quad-W_{e}^{2}\left(1-a^{2}\right)\left(\frac{d^{2} v}{\mathrm{~d} x^{2}}+\varphi \frac{d^{2} v}{\mathrm{~d} x^{2}}-2 S_{t}\right)\left(\frac{\mathrm{d} v}{\mathrm{~d} x}\right)^{2}+S_{t}=0 .
\end{aligned}
$$

Three cases as fractional boundary value problems are obtained. 
TABLE 1: Solution and residual error for $\alpha$ keeping $a=0.1, W_{e}=0.01, S_{t}=0.001$, and $\phi=0.1$ fixed in lifting.

\begin{tabular}{ccccccc}
\hline & \multicolumn{3}{c}{$\alpha=0.2$} & \multicolumn{2}{c}{$\alpha=0.6$} & \multicolumn{2}{c}{$\alpha=0.99$} \\
$x$ & $V(x)$ & Res error & $V(x)$ & Res error & $(x)$ & 0.905 \\
\hline 0.1 & 0.905 & $-8.76128 \times 10^{-25}$ & 0.905 & $-9.08606 \times 10^{-24}$ & $-6.59422 \times 10^{-23}$ \\
0.2 & 0.82 & $-2.5981 \times 10^{-24}$ & 0.82 & $-1.45747 \times 10^{-23}$ & 0.82 & $-4.249 \times 10^{-23}$ \\
0.3 & 0.745 & $-4.78151 \times 10^{-24}$ & 0.745 & $-1.74003 \times 10^{-23}$ & 0.745 & $-2.54931 \times 10^{-23}$ \\
0.4 & 0.68 & $-7.11425 \times 10^{-24}$ & 0.68 & $-1.79924 \times 10^{-23}$ & 0.68 & $-1.41813 \times 10^{-24}$ \\
0.5 & 0.625 & $-9.29068 \times 10^{-24}$ & 0.625 & $-1.69071 \times 10^{-23}$ & 0.625 & $-6.9968 \times 10^{-24}$ \\
0.6 & 0.58 & $-1.10441 \times 10^{-23}$ & 0.58 & $-1.4743 \times 10^{-23}$ & 0.58 & $-3.0318 \times 10^{-24}$ \\
0.7 & 0.545 & $-1.2179 \times 10^{-23}$ & 0.545 & $-1.20434 \times 10^{-23}$ & 0.545 & $-1.0542 \times 10^{-24}$ \\
0.8 & 0.52 & $-1.25923 \times 10^{-23}$ & 0.52 & $-9.26204 \times 10^{-24}$ & 0.52 & $-2.52839 \times 10^{-25}$ \\
0.9 & 0.505 & $-1.22773 \times 10^{-23}$ & 0.505 & $-6.71129 \times 10^{-24}$ & 0.505 & $-2.91563 \times 10^{-27}$ \\
1. & 0.5 & $-1.13159 \times 10^{-23}$ & 0.5 & $-4.57323 \times 10^{-24}$ & 0.5 & $1.0211 \times 10^{-26}$ \\
\hline
\end{tabular}

TABLE 2: Solution and residual error for $\phi$ keeping $\alpha=0.99, W_{e}=0.01, S_{t}=0.001$, and $a=0.1$ fixed in lifting.

\begin{tabular}{|c|c|c|c|c|c|c|}
\hline \multirow[b]{2}{*}{$x$} & \multicolumn{2}{|c|}{$\phi=0.1$} & \multicolumn{2}{|c|}{$\phi=0.6$} & \multicolumn{2}{|c|}{$\phi=0.9$} \\
\hline & $V(x)$ & Res error & $V(x)$ & Res error & $V(x)$ & Res error \\
\hline 0.1 & 0.905 & $-6.59422 \times 10^{-23}$ & 0.905 & $-9.09923 \times 10^{-23}$ & 0.905 & $-1.08443 \times 10-22$ \\
\hline 0.2 & 0.82 & $-4.249 \times 10^{-23}$ & 0.82 & $-5.8628 \times 10^{-23}$ & 0.82 & $-6.98245 \times 10^{-23}$ \\
\hline 0.3 & 0.745 & $-2.54931 \times 10^{-23}$ & 0.745 & $-3.51797 \times 10^{-23}$ & 0.745 & $-4.19438 \times 10^{-23}$ \\
\hline 0.4 & 0.68 & $-1.41813 \times 10^{-23}$ & 0.68 & $-1.95548 \times 10^{-23}$ & 0.68 & $-2.32819 \times 10^{-23}$ \\
\hline 0.5 & 0.625 & $-6.9968 \times 10^{-24}$ & 0.625 & $-9.6702 \times 10^{-24}$ & 0.625 & $-1.15297 \times 10^{-23}$ \\
\hline 0.6 & 0.58 & $-3.0318 \times 10^{-24}$ & 0.58 & $-4.18483 \times 10^{-24}$ & 0.58 & $-4.96745 \times 10^{-24}$ \\
\hline 0.7 & 0.545 & $-1.0542 \times 10^{-24}$ & 0.545 & $-1.45024 \times 10^{-24}$ & 0.545 & $-1.7253 \times 10^{-24}$ \\
\hline 0.8 & 0.52 & $-2.52839 \times 10^{-25}$ & 0.52 & $-3.53713 \times 10^{-25}$ & 0.52 & $-4.20658 \times 10^{-25}$ \\
\hline 0.9 & 0.505 & $-2.91563 \times 10^{-27}$ & 0.505 & $-1.76326 \times 10^{-26}$ & 0.505 & $-2.24163 \times 10^{-26}$ \\
\hline 1. & 0.5 & $1.0211 \times 10^{-26}$ & 0.5 & $5.3895 \times 10^{-27}$ & 0.5 & $9.2139 \times 10^{-27}$ \\
\hline
\end{tabular}

TABLE 3: Solution and residual error for $S_{t}$ keeping $\alpha=0.98, W_{e}=0.001, \phi=0.1$, and $a=0.1$ fixed in lifting.

\begin{tabular}{ccccccc}
\hline & \multicolumn{2}{c}{$S_{t}=0.001$} & \multicolumn{2}{c}{$S_{t}=0.01$} & \multicolumn{2}{c}{$S_{t}=0.1$} \\
$x$ & $V(x)$ & Res error & $V(x)$ & Res error & $V(x)$ & $-6.30818 \times 10^{-17}$ \\
\hline 0.1 & 0.999905 & $-7.23543 \times 10^{-27}$ & 0.99905 & $-6.29219 \times 10^{-22}$ & 0.9905 & $-4.18849 \times 10^{-17}$ \\
0.2 & 0.99982 & $-3.63823 \times 10^{-27}$ & 0.9982 & $-4.18657 \times 10^{-22}$ & 0.982 & $-2.57976 \times 10^{-17}$ \\
0.3 & 0.999745 & $-2.86435 \times 10^{-27}$ & 0.99745 & $-2.5807 \times 10^{-22}$ & 0.9745 & $-1.46541 \times 10^{-18}$ \\
0.4 & 0.99968 & $-1.51698^{*} \times 10^{-27}$ & 0.9968 & $-1.46438 \times 10^{-22}$ & 0.968 & $-7.52476 \times 10^{-18}$ \\
0.5 & 0.999625 & $-8.70705 \times 10^{-28}$ & 0.99625 & $-7.53253 \times 10^{-23}$ & 0.9625 & $-3.37798 \times 10^{-18}$ \\
0.6 & 0.99958 & $-4.61286 \times 10^{-28}$ & 0.9958 & $-3.40645 \times 10^{-23}$ & 0.958 & $-1.25942 \times 10^{-18}$ \\
0.7 & 0.999545 & $-4.34662 \times 10^{-28}$ & 0.99545 & $-1.28478 \times 10^{-23}$ & 0.9545 & $-3.64878 \times 10^{-19}$ \\
0.8 & 0.99952 & $-4.00347 \times 10^{-29}$ & 0.9952 & $-3.70343 \times 10^{-24}$ & 0.952 & $-8.14999 \times 10^{-20}$ \\
0.9 & 0.999505 & $-1.66647 \times 10^{-29}$ & 0.99505 & $-1.07941 \times 10^{-24}$ & 0.9505 & -8.145 \\
1. & 0.9995 & $9.95937 \times 10^{-30}$ & 0.995 & $-8.78476 \times 10^{-26}$ & 0.95 & $-8.71776 \times 10^{-21}$ \\
\hline
\end{tabular}

TABLE 4: Solution and residual error for $W_{e}$ keeping $\alpha=0.95, S_{t}=0.001, \phi=0.1$, and $a=0.1$ fixed in lifting.

\begin{tabular}{|c|c|c|c|c|c|c|}
\hline \multirow[b]{2}{*}{$x$} & \multicolumn{2}{|c|}{$W_{e}=0.001$} & \multicolumn{2}{|c|}{$W_{e}=0.01$} & \multicolumn{2}{|c|}{$W_{e}=0.1$} \\
\hline & $V(x)$ & Res error & $V(x)$ & Res error & $V(x)$ & Res error \\
\hline 0.1 & 0.905 & $-5.48732 \times 10^{-27}$ & 0.905 & $-5.51525 \times 10^{-23}$ & 0.905 & $-5.4982 \times 10^{-19}$ \\
\hline 0.2 & 0.82 & $-3.75182 \times 10^{-27}$ & 0.82 & $-3.98956 \times 10^{-23}$ & 0.82 & $-3.98946 \times 10^{-19}$ \\
\hline 0.3 & 0.745 & $-2.5638 \times 10^{-27}$ & 0.745 & $-2.63606 \times 10^{-23}$ & 0.745 & $-2.63525 \times 10^{-19}$ \\
\hline 0.4 & 0.68 & $-1.17028 \times 10^{-27}$ & 0.68 & $-1.60262 \times 10^{-23}$ & 0.68 & $-1.6049 \times 10^{-19}$ \\
\hline 0.5 & 0.625 & $-7.01495 \times 10^{-28}$ & 0.625 & $-8.9237 \times 10^{-24}$ & 0.625 & $-8.93613 \times 10^{-20}$ \\
\hline 0.6 & 0.58 & $-4.80416 \times 10^{-28}$ & 0.58 & $-4.47664 \times 10^{-24}$ & 0.58 & $-4.46647 \times 10^{-20}$ \\
\hline 0.7 & 0.545 & $-3.2225 \times 10^{-28}$ & 0.545 & $-1.95569 \times 10^{-24}$ & 0.545 & $-1.9563 \times 10^{-20}$ \\
\hline 0.8 & 0.52 & $7.79 \times 10^{-30}$ & 0.52 & $-7.49229 \times 10^{-25}$ & 0.52 & $-7.37223 \times 10^{-21}$ \\
\hline 0.9 & 0.505 & $1.3884 \times 10^{-28}$ & 0.505 & $-2.49356 \times 10^{-25}$ & 0.505 & $-2.42499 \times 10^{-21}$ \\
\hline 1. & 0.5 & $9.40717 \times 10^{-29}$ & 0.5 & $-6.13229 \times 10^{-26}$ & 0.5 & $-5.63892 \times 10^{-22}$ \\
\hline
\end{tabular}


TABLE 5: Solution and residual error for $\alpha$ keeping $a=0.1, W_{e}=0.01, S_{t}=0.001$, and $\phi=0.1$ fixed in Case 1 of drainage.

\begin{tabular}{ccccccc}
\hline & \multicolumn{2}{c}{$\alpha=0.3$} & \multicolumn{2}{c}{$\alpha=0.6$} & \multicolumn{2}{c}{$\alpha=0.9$} \\
$x$ & $V(x)$ & Res error & $V(x)$ & Res error & $V(x)$ & \multicolumn{2}{c}{ Res error } \\
\hline 0.1 & 0.000095 & $1.61655 \times 10^{-24}$ & 0.000095 & $9.08344 \times 10^{-24}$ & 0.000095 & $6.30751 \times 10^{-23}$ \\
0.2 & 0.00018 & $4.16166 \times 10^{-24}$ & 0.00018 & $1.45689 \times 10^{-23}$ & 0.00018 & $4.18362 \times 10^{-23}$ \\
0.3 & 0.000255 & $6.96312 \times 10^{-24}$ & 0.000255 & $1.73923 \times 10^{-23}$ & 0.000255 & $2.57623 \times 10^{-23}$ \\
0.4 & 0.00032 & $9.57269 \times 10^{-24}$ & 0.00032 & $1.79837 \times 10^{-23}$ & 0.00032 & $1.46516 \times 10^{-23}$ \\
0.5 & 0.000375 & $1.16444 \times 10^{-23}$ & 0.000375 & $1.6899 \times 10^{-23}$ & 0.000375 & $7.51521 \times 10^{-24}$ \\
0.6 & 0.00042 & $1.29537 \times 10^{-23}$ & 0.00042 & $1.47362 \times 10^{-23}$ & 0.00042 & $3.40636 \times 10^{-24}$ \\
0.7 & 0.000455 & $1.34057 \times 10^{-23}$ & 0.000455 & $1.20384 \times 10^{-23}$ & 0.000455 & $1.25929 \times 10^{-24}$ \\
0.8 & 0.00048 & $1.30281 \times 10^{-23}$ & 0.00048 & $9.25865 \times 10^{-24}$ & 0.00048 & $3.70727 \times 10^{-25}$ \\
0.9 & 0.000495 & $1.19467 \times 10^{-23}$ & 0.000495 & $6.70931 \times 10^{-24}$ & 0.000495 & $8.09182 \times 10^{-26}$ \\
1. & 0.0005 & $1.0354 \times 10^{-23}$ & 0.0005 & $4.57227 \times 10^{-24}$ & 0.0005 & $1.89895 \times 10^{-26}$ \\
\hline
\end{tabular}

TABLE 6: Solution and residual error for $\phi$ keeping $\alpha=0.98, W_{e}=0.01, S_{t}=0.001$, and $a=0.1$ fixed for Case 1 of drainage.

\begin{tabular}{ccccccc}
\hline & \multicolumn{2}{c}{$\phi=0.01$} & \multicolumn{2}{c}{$\phi=0.1$} & \multicolumn{2}{c}{$\phi=0.3$} \\
$x$ & $V(x)$ & Res error & $V(x)$ & Res error & $V(x)$ & 0.000095 \\
\hline 0.1 & 0.000095 & $5.92844 \times 10^{-23}$ & 0.000095 & $6.30751 \times 10^{-23}$ & $8.18541 \times 10^{-23}$ \\
0.2 & 0.00018 & $3.93158 \times 10^{-23}$ & 0.00018 & $4.18362 \times 10^{-23}$ & 0.00018 & $5.42952 \times 10^{-23}$ \\
0.3 & 0.000255 & $2.42117 \times 10^{-23}$ & 0.000255 & $2.57623 \times 10^{-23}$ & 0.000255 & $3.34316 \times 10^{-23}$ \\
0.4 & 0.00032 & $1.37711 \times 10^{-23}$ & 0.00032 & $1.46516 \times 10^{-23}$ & 0.00032 & $1.90143 \times 10^{-23}$ \\
0.5 & 0.000375 & $7.0636 \times 10^{-24}$ & 0.000375 & $7.51521 \times 10^{-24}$ & 0.000375 & $9.74926 \times 10^{-24}$ \\
0.6 & 0.00042 & $3.20361 \times 10^{-24}$ & 0.00042 & $3.40636 \times 10^{-24}$ & 0.00042 & $4.41229 \times 10^{-24}$ \\
0.7 & 0.000455 & $1.18469 \times 10^{-24}$ & 0.000455 & $1.25929 \times 10^{-24}$ & 0.000455 & $1.62912 \times 10^{-24}$ \\
0.8 & 0.00048 & $3.49409 \times 10^{-25}$ & 0.00048 & $3.70727 \times 10^{-25}$ & 0.00048 & $4.82038 \times 10^{-25}$ \\
0.9 & 0.000495 & $7.66394 \times 10^{-26}$ & 0.000495 & $8.09182 \times 10^{-26}$ & 0.000495 & $1.05556 \times 10^{-25}$ \\
1. & 0.0005 & $1.87118 \times 10^{-26}$ & 0.0005 & $1.89895 \times 10^{-26}$ & 0.0005 & $2.15706 \times 10^{-24}$ \\
\hline
\end{tabular}

TABLE 7: Solution and residual error for $S_{t}$ keeping $\alpha=0.99, W_{e}=0.01, \phi=0.01$, and $a=0.1$ fixed in Case 1 of drainage.

\begin{tabular}{ccccccc}
\hline & \multicolumn{2}{c}{$S_{t}=0.001$} & \multicolumn{2}{c}{$S_{t}=0.01$} & \multicolumn{2}{c}{$S_{t}=0.1$} \\
$x$ & $V(x)$ & Res error & $V(x)$ & Res error & $V(x)$ & $5.8252 \times 10--13$ \\
0.1 & 0.000095 & $5.82872 \times 10^{-23}$ & 0.000095 & $5.82511 \times 10^{-18}$ & 0.000095 & $3.75135 \times 10--13$ \\
0.2 & 0.00018 & $3.7525 \times 10^{-23}$ & 0.00018 & $3.75136 \times 10^{-18}$ & 0.00018 & $2.25477 \times 10--13$ \\
0.3 & 0.000255 & $2.24807 \times 10^{-23}$ & 0.000255 & $2.25476 \times 10^{-18}$ & 0.000255 & $1.2492 \times 10--13$ \\
0.4 & 0.00032 & $1.25172 \times 10^{-23}$ & 0.00032 & $1.24919 \times 10^{-18}$ & 0.00032 & $6.22317 \times 10--14$ \\
0.5 & 0.000375 & $6.177 \times 10^{-24}$ & 0.000375 & $6.22301 \times 10^{-19}$ & 0.000375 & 0.00042 \\
0.6 & 0.00042 & $2.69667 \times 10^{-24}$ & 0.00042 & $2.67581 \times 10^{-19}$ & 0.000455 & $9.2681 \times 10--15$ \\
0.7 & 0.000455 & $9.14662 \times 10^{-25}$ & 0.000455 & $9.26852 \times 10^{-20}$ & 0.00048 & $2.3095 \times 10--15$ \\
0.8 & 0.00048 & $2.38577 \times 10^{-25}$ & 0.00048 & $2.30937 \times 10^{-20}$ & 0.000495 & $3.76414 \times 10--16$ \\
0.9 & 0.000495 & $2.25046 \times 10^{-26}$ & 0.000495 & $3.77223 \times 10^{-21}$ & 0.005 & $1.88184 \times 10^{-17}$ \\
1. & 0.0005 & $3.98217 \times 10^{-27}$ & 0.0005 & $1.84694 \times 10^{-22}$ & 0.0005 \\
\hline
\end{tabular}

TABLE 8: Solution and residual error for $\gamma$ keeping $S_{t}=0.01, \phi=0.1, W_{e}=0.001$, and $a=0.1$ fixed in Case 2 of drainage.

\begin{tabular}{ccccccc}
\hline & \multicolumn{2}{c}{$\gamma=1.2$} & \multicolumn{2}{c}{$\gamma=1.6$} & \multicolumn{2}{c}{$\gamma=1.99$} \\
$x$ & $V(x)$ & Res error & $V(x)$ & Res error & $V(x)$ & $1.29417 \times 10^{-17}$ \\
\hline 0.1 & $5.72659 \times 10--4$ & $-1.32727 \times 10^{-18}$ & $1.75703 \times 10--4$ & $3.197 \times 10^{-18}$ & $5.1638 \times 10--5$ \\
0.2 & $1.31563 \times 10--3$ & $-2.02422 \times 10^{-18}$ & $5.3263 \times 10--3$ & $3.56035 \times 10^{-18}$ & $2.05125 \times 10--4$ & $1.22623 \times 10^{-17}$ \\
0.3 & $2.14014 \times 10--3$ & $-1.98946 \times 10^{-18}$ & $1.01899 \times 10--3$ & $3.88346 \times 10^{-18}$ & $4.59664 \times 10--4$ & $1.24389 \times 10^{-17}$ \\
0.4 & $3.02251 \times 10--3$ & $-2.9885 \times 10^{-19}$ & $1.61463 \times 10--3$ & $3.46852 \times 10^{-18}$ & $8.14833 \times 10--4$ & $1.35961 \times 10^{-17}$ \\
0.5 & $3.95057 \times 10--3$ & $-1.84225 \times 10^{-18}$ & $2.30744 \times 10--3$ & $3.68767 \times 10^{-18}$ & $1.27034 \times 10--3$ & $1.31157 \times 10^{-17}$ \\
0.6 & $4.91675 \times 10--3$ & $-2.46976 \times 10^{-18}$ & $3.08901 \times 10--3$ & $3.57668 \times 10^{-18}$ & $1.82595 \times 10--3$ & $1.37891 \times 10^{-17}$ \\
0.7 & $5.91581 \times 10--3$ & $-2.09831 \times 10^{-18}$ & $3.95307 \times 10--3$ & $3.36789 \times 10^{-18}$ & $2.4815 \times 10--3$ & $1.30025 \times 10^{-17}$ \\
0.8 & $6.94391 \times 10--3$ & $-1.35899 \times 10^{-18}$ & $4.89465 \times 10--3$ & $3.06616 \times 10^{-18}$ & $3.23682 \times 10--3$ & $1.40449 \times 10^{-17}$ \\
0.9 & $7.99811 \times 10--3$ & $-5.26745 \times 10^{-19}$ & $5.90971 \times 10--3$ & $4.003 \times 10^{-18}$ & $4.09177 \times 10--3$ & $1.29653 \times 10^{-17}$ \\
1. & $9.07604 \times 10--3$ & $-1.50223 \times 10^{-18}$ & $6.99484 \times 10--3$ & $2.56045 \times 10^{-18}$ & $5.04625 \times 10--3$ & $1.32103 \times 10^{-17}$ \\
\hline
\end{tabular}


TABLE 9: Solution and residual error for $\alpha$ keeping $\gamma=1.95, S_{t}=0.001, \phi=0.1, W_{e}=0.01$, and $a=0.1$ fixed in Case 3 of drainage.

\begin{tabular}{ccccccc}
\hline & \multicolumn{2}{c}{$\alpha=0.3$} & \multicolumn{2}{c}{$\alpha=0.7$} & \multicolumn{2}{c}{$\alpha=0.99$} \\
$x$ & $V(x)$ & Res error & $V(x)$ & Res error & $V(x)$ & Res error \\
\hline 0.1 & $5.87208 \times 10--6$ & $-9.076 \times 10^{-20}$ & $5.87208 \times 10--6$ & $1.16701 \times 10^{-19}$ & $5.87208 \times 10--6$ & $6.48938 \times 10^{-21}$ \\
0.2 & $2.26882 \times 10--5$ & $6.62623 \times 10^{-20}$ & $2.26882 \times 10--5$ & $-3.50514 \times 10^{-20}$ & $2.26882 \times 10--5$ & $-1.64872 \times 10^{-19}$ \\
0.3 & $5.0024 \times 10--5$ & $7.16925 \times 10^{-20}$ & $5.0024 \times 10--5$ & $-5.38787 \times 10^{-20}$ & $5.0024 \times 10--5$ & $-5.48153 \times 10^{-20}$ \\
0.4 & $8.76616 \times 10--5$ & $6.17061 \times 10^{-20}$ & $8.76616 \times 10--5$ & $4.81294 \times 10^{-20}$ & $8.76616 \times 10--5$ & $-2.07791 \times 10^{-19}$ \\
0.5 & $1.35451 \times 10--4$ & $-7.89358 \times 10^{-20}$ & $1.35451 \times 10--4$ & $8.88535 \times 10^{-20}$ & $1.35451 \times 10--4$ & $6.49314 \times 10^{-20}$ \\
0.6 & $1.9328 \times 10--4$ & $5.62103 \times 10^{-20}$ & $1.9328 \times 10--4$ & $-8.1769 \times 10^{-20}$ & $1.9328 \times 10--4$ & $-1.40755 \times 10^{-20}$ \\
0.7 & $2.61056 \times 10--4$ & $2.13652 \times 10^{-20}$ & $2.61056 \times 10--4$ & $2.46311 \times 10^{-19}$ & $2.61056 \times 10--4$ & $8.01669 \times 10^{-21}$ \\
0.8 & $3.38702 \times 10--4$ & $4.3926 \times 10^{-20}$ & $3.38702 \times 10--4$ & $-3.74249 \times 10^{-20}$ & $3.38702 \times 10--4$ & $-1.55483 \times 10^{-19}$ \\
0.9 & $4.26153 \times 10--4$ & $-1.25871 \times 10^{-19}$ & $4.26153 \times 10--4$ & $-4.78429 \times 10^{-20}$ & $4.26153 \times 10--4$ & $-1.57049 \times 10^{-19}$ \\
1. & $5.2335 \times 10--4$ & $7.27423 \times 10^{-20}$ & $5.2335 \times 10--4$ & $3.94566 \times 10^{-20}$ & $5.2335 \times 10--4$ & $-5.88772 \times 10^{-20}$ \\
\hline
\end{tabular}

TABLE 10: Solution and residual error for $\gamma$ keeping $\alpha=0.95, S_{t}=0.001, \phi=0.1, W_{e}=0.01$, and $a=0.1$ fixed in Case 3 of drainage.

\begin{tabular}{ccccccc}
\hline & \multicolumn{2}{c}{$\gamma=1.2$} & \multicolumn{2}{c}{$\gamma=1.6$} & \multicolumn{2}{c}{$\gamma=1.99$} \\
$x$ & $V(x)$ & Res error & $V(x)$ & Res error & $V(x)$ & Res error \\
\hline 0.1 & $5.72659 \times 10--4$ & $-8.85316 \times 10^{-20}$ & $1.75703 \times 10--5$ & $-9.8013 \times 10^{-20}$ & $5.1638 \times 10--6$ & $4.95955 \times 10^{-21}$ \\
0.2 & $1.31563 \times 10--4$ & $-1.35853 \times 10^{-19}$ & $5.3263 \times 10--5$ & $-5.72227 \times 10^{-20}$ & $2.0512 \times 10--5$ & $1.06505 \times 10^{-20}$ \\
0.3 & $2.14014 \times 10--4$ & $1.63841 \times 10^{-20}$ & $1.01899 \times 10--4$ & $3.02498 \times 10^{-22}$ & $4.5966 \times 10--5$ & $2.98796 \times 10^{-20}$ \\
0.4 & $3.02251 \times 10--4$ & $-1.77002 \times 10^{-19}$ & $1.61463 \times 10--4$ & $2.74106 \times 10^{-19}$ & $8.1483 \times 10--5$ & $8.85672 \times 10^{-20}$ \\
0.5 & $3.95057 \times 10--4$ & $6.75864 \times 10^{-20}$ & $2.30744 \times 10--4$ & $-2.9077 \times 10^{-20}$ & $1.2703 \times 10--4$ & $-1.25093 \times 10^{-19}$ \\
0.6 & $4.91675 \times 10--4$ & $-1.24341 \times 10^{-19}$ & $3.08901 \times 10--4$ & $2.8962 \times 10^{-19}$ & $1.8259 \times 10--4$ & $7.08777 \times 10^{-21}$ \\
0.7 & $5.91581 \times 10--4$ & $3.33152 \times 10^{-20}$ & $3.95307 \times 10--4$ & $4.4664 \times 10^{-20}$ & $2.4815 \times 10--4$ & $3.73471 \times 10^{-20}$ \\
0.8 & $6.94391 \times 10--4$ & $-5.4743 \times 10^{-20}$ & $4.89465 \times 10--4$ & $-4.42716 \times 10^{-20}$ & $3.2368 \times 10--4$ & $-2.48835 \times 10^{-20}$ \\
0.9 & $7.99811 \times 10--4$ & $-4.85706 \times 10^{-20}$ & $5.90971 \times 10--4$ & $-1.45847 \times 10^{-19}$ & $4.0917 \times 10--4$ & $1.10885 \times 10^{-19}$ \\
1. & $9.07604 \times 10--4$ & $-2.09174 \times 10^{-19}$ & $6.99484 \times 10--4$ & $1.98754 \times 10^{-20}$ & $5.0462 \times 10--4$ & $2.57162 \times 10^{-19}$ \\
\hline
\end{tabular}

Case 1

$$
\begin{aligned}
& \frac{d^{2} v(x)}{\mathrm{d} x^{2}}+W_{e}^{4}\left(1-a^{2}\right)^{2}\left[\varphi \frac{d^{2} v(x)}{\mathrm{d} x^{2}}+S_{t}\right]\left(D^{\alpha} v(x)\right)^{4} \\
& -W_{e}^{2}\left(1-a^{2}\right)\left[\frac{d^{2} v(x)}{\mathrm{d} x^{2}}+\varphi \frac{d^{2} v(x)}{\mathrm{d} x^{2}}-2 S_{t}\right]\left(D^{\alpha} v(x)\right)^{2}+S_{t}=0,
\end{aligned}
$$

with

$$
v(0)=0, v^{\prime}(1)=0,0<\alpha<1
$$

Case 2

$$
\begin{aligned}
& D^{\gamma} v(x)+W_{e}^{4}\left(1-a^{2}\right)^{2}\left[\varphi D^{\gamma} v(x)+S_{t}\right]\left(\frac{\mathrm{d} v(x)}{\mathrm{d} x}\right)^{4} \\
& -W_{e}^{2}\left(1-a^{2}\right)\left[D^{\gamma} v(x)+D^{\gamma} v(x)-2 S_{t}\right]\left(\frac{\mathrm{d} v(x)}{\mathrm{d} x}\right)^{2}+S_{t}=0 . \\
& \quad v(0)=0, v^{\prime}(1)=0,1<\gamma<2 .
\end{aligned}
$$

Case 3. Here both first- and second-order derivatives are replaced by the noninteger order derivative as follows:

$$
\begin{aligned}
& D^{\gamma} v(x)+W_{e}^{4}\left(1-a^{2}\right)^{2}\left[\varphi D^{\gamma} v(x)+S_{t}\right]\left(D^{\alpha} v(x)\right)^{4} \\
& \quad-W_{e}^{2}\left(1-a^{2}\right)\left[D^{\gamma} v(x)+D^{\gamma} v(x)-2 S_{t}\right]\left(D^{\alpha} v(x)\right)^{2}+S_{t}=0,
\end{aligned}
$$

with

$$
v(0)=0, v(1)=0,1<\gamma<2,0<\alpha<1 .
$$

\section{Results and Discussion}

In this paper, the fractional study of thin film flow of Johnson-Segalman fluid is carried out in lifting and drainage cases. The obtained boundary value problems are solved for different values of involved parameters and results mentioned in Tables 1-4 for lifting while Tables 5-10 in drainage situation. Tables 1 and 5 present solutions and corresponding errors for different $\alpha$. Tables 2 and 6 show solutions along with errors for $\phi$. Tables 3 and 7 show case solutions and errors for various $S_{t}$. Table 4 demonstrates solutions and errors for various $W_{e}$ in the lifting case.

Tables 8-10 exhibit solutions and residual errors for different values of fractional parameters in Cases 2 and 3, respectively. Exploration of these tables confirms that solutions are consistent. Influence of different parameters on $V(x)$ in lifting and drainage cases are seen graphically. Figures $1-8$ show the effect of different parameters on $V(x)$ 


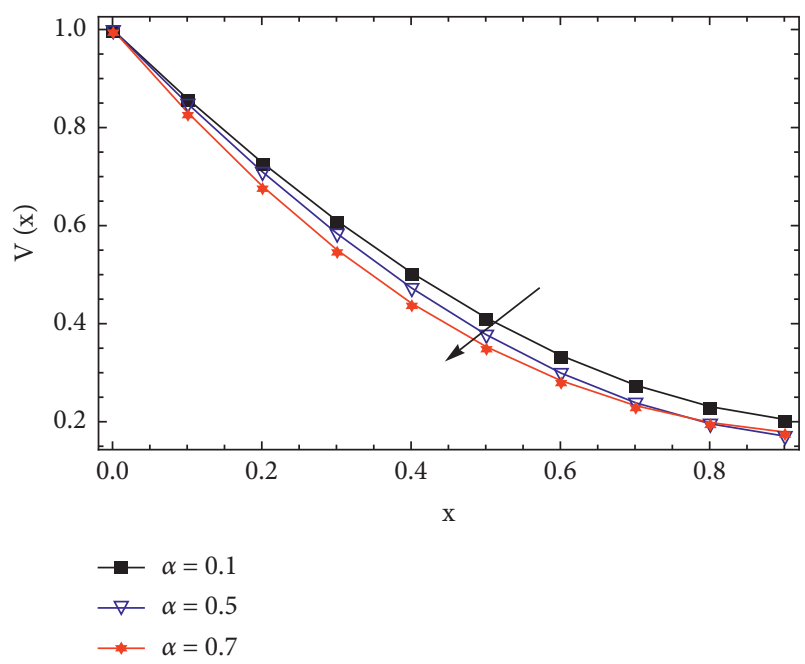

Figure 1: Effect of $\alpha$ on $V(x)$ for $a=0.1, W_{e}=1, S_{t}=1$, and $\phi=$ 0.1 fixed in the lifting case.

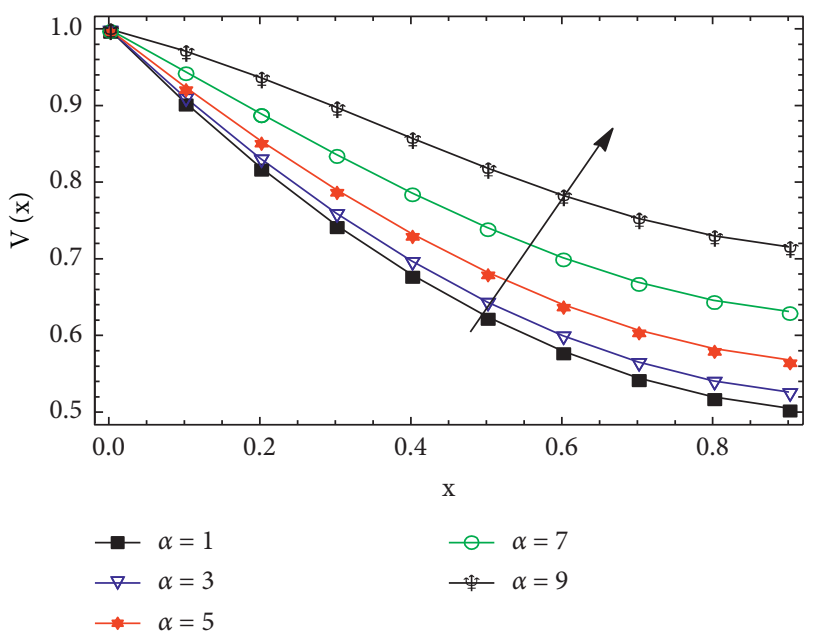

Figure 2: Effect of $a$ on $V(x)$ for $\alpha=0.95, S_{t}=1, \phi=$ 0.01 , and $W_{e}=0.1$ fixed in the lifting case.

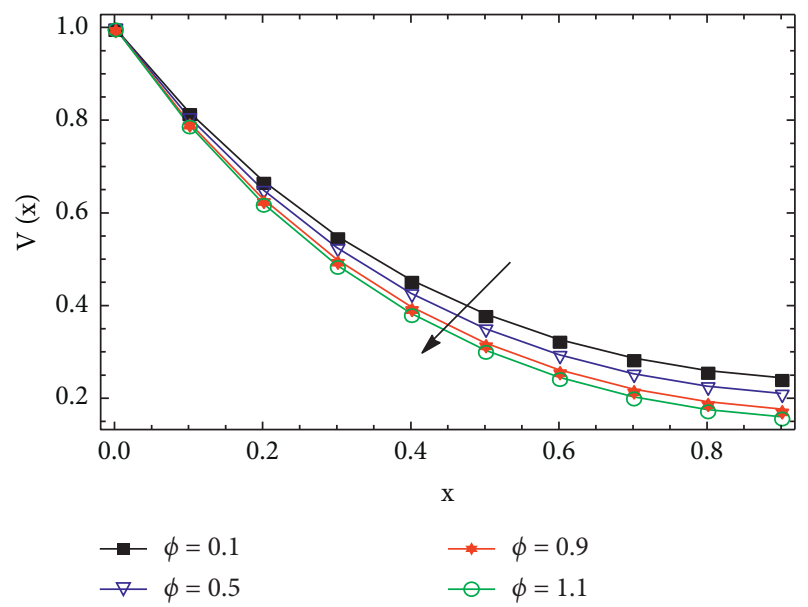

Figure 3: Effect of $\phi$ on $V(x)$ for $\alpha=0.98, a=0.1$, $W_{e}=1$, and $S_{t}=1$ fixed in the lifting case.

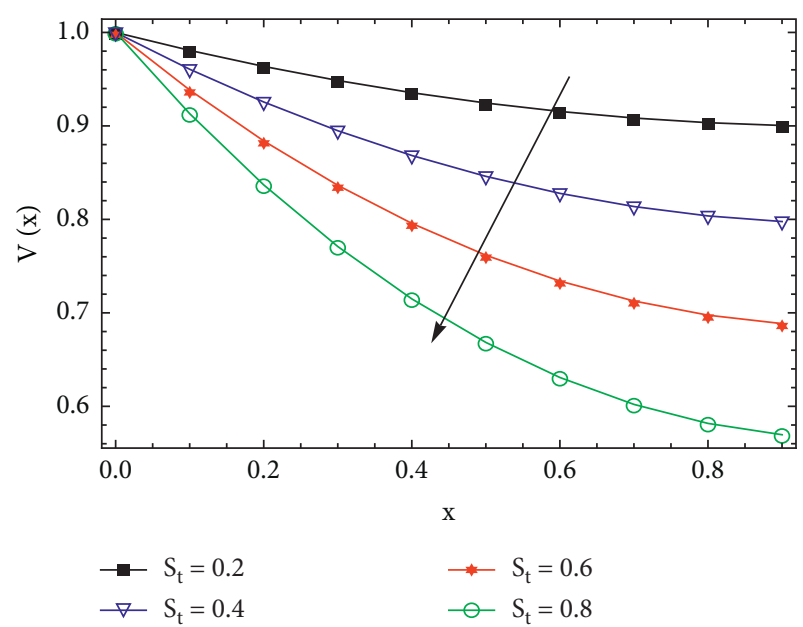

FIgURE 4: The effect of $S_{t}$ on $V(x)$ for $\alpha=0.95, a=0.1, W_{e}=$ 1 , and $\phi=0.1$ fixed in the lifting case.

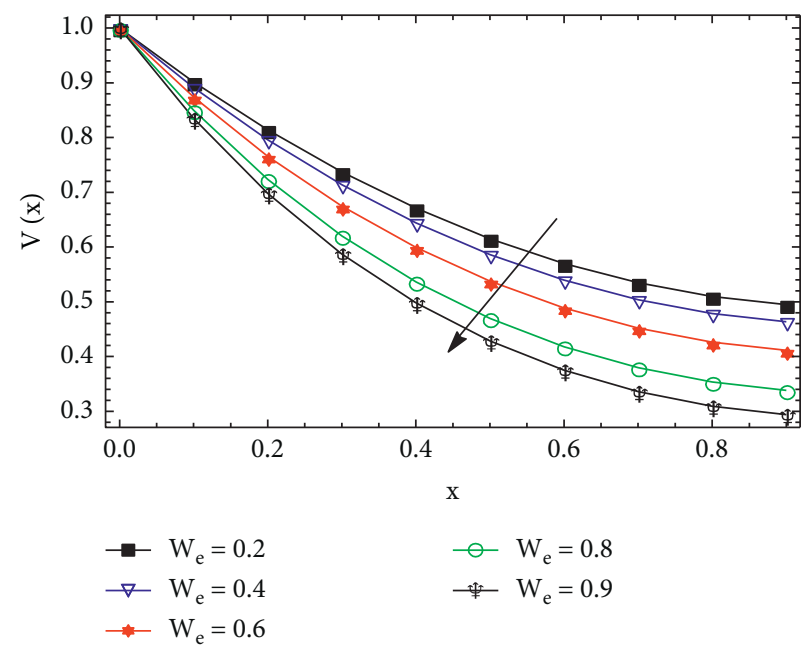

Figure 5: Effect of $W_{e}$ on $V(x)$ for $\alpha=0.98, a=$ $0.1, S_{t}=1$, and $\phi=0.1$ fixed in the lifting case.

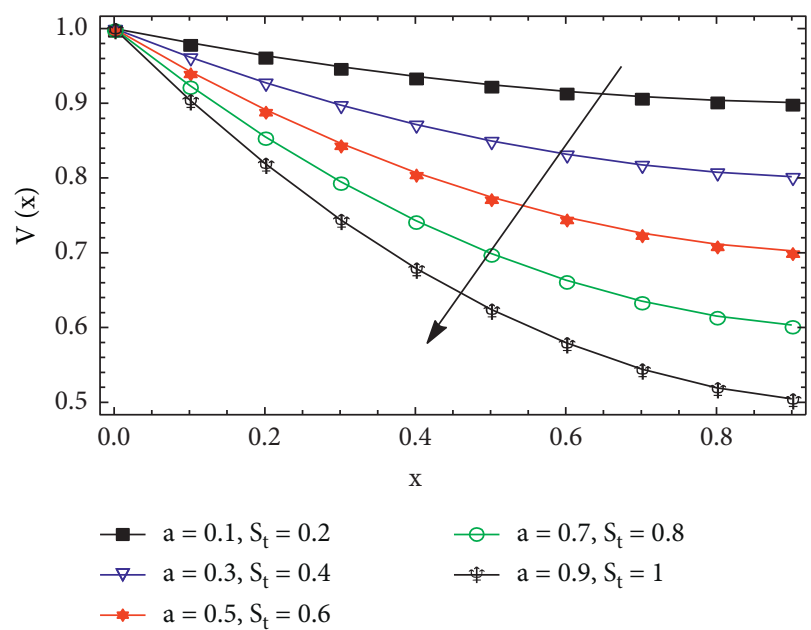

FIGURE 6: Effect of increasing $S_{t}$ and $a$ simultaneously on $V(x)$ for $\alpha=0.99, W_{e}=0.1$, and $\phi=0.1$ fixed in the lifting case. 


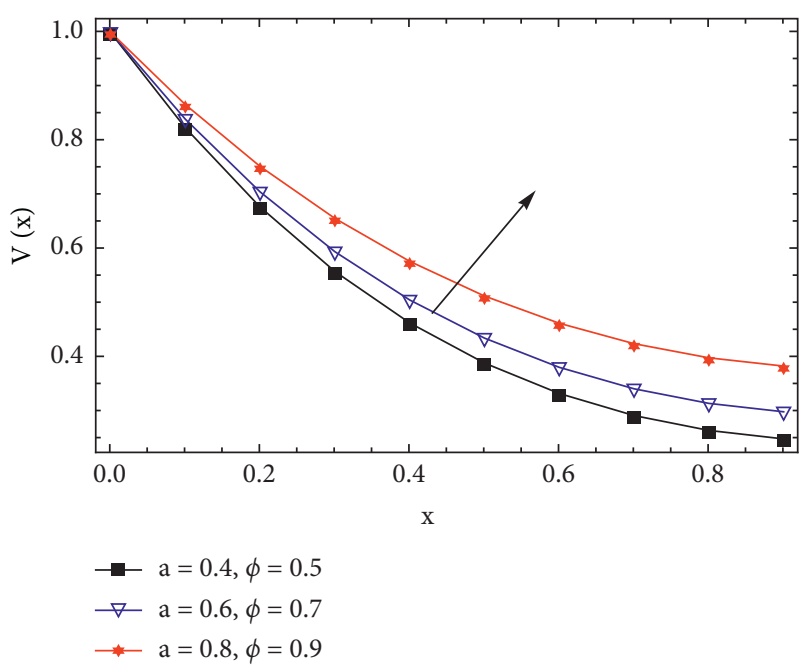

FIgURE 7: Effect of increasing $\phi$ and $a$ simultaneously on $V(x)$ for $\alpha=0.95, W_{e}=1, S_{t}=0.1$ fixed in the lifting case.

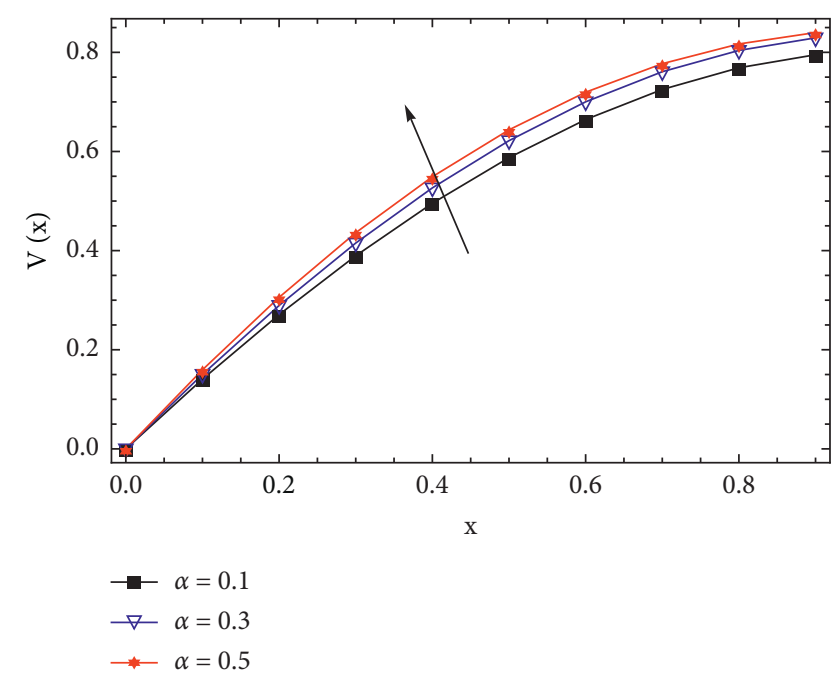

FIgURE 8: Effect of $\alpha$ on $V(x)$ for $a=0.1, W_{e}=1, S_{t}=1$, and $\phi=$ 0.1 fixed for Case 1 in the drainage case.

in the lifting case. Figure 1 shows the effect of $\alpha$ on $V(x)$. It is seen that increasing $\alpha$ reduces $V(x)$. Figure 2 shows effect of $a$ on $V(x)$, showing that $a$ has direct relationship with $V(x)$. Figures 3-5 depict the consequence of $\phi, S_{t}$, and $W_{e}$ on $V(x)$, respectively. It is observed that Weissenberg number is the dimensionless number while this parameter is used to measure the flow behavior into motion regarding particles. It is physically defined as ratio among viscous force and elastic force. Viscous force is enhanced against higher values of Weissenberg number, whereas higher viscous force brings a declination into motion of fluid particles. Momentum layers are also the reducing function versus implanted higher values of the Weissenberg number. It is seen that $V(x)$ has inverse relationship with mentioned parameters in all cases. Figure 6 indicates the impact of increasing $a$ and $S_{t}$ simultaneously on $V(x)$. It is observed that $V(x)$ decreases with an increase in $a$ and $S_{t}$, and hence, $S_{t}$ is a more

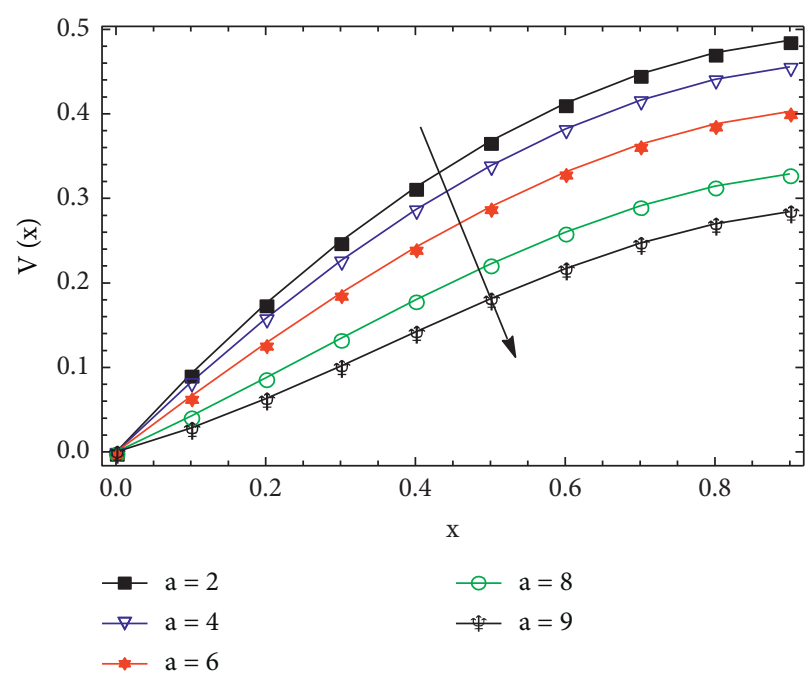

FIgURE 9: Effect of $a$ on $V(x)$ for $\alpha=0.95, W_{e}=0.1$, $S_{t}=1$, and $\phi=0.01$ fixed for Case 1 in the drainage case.

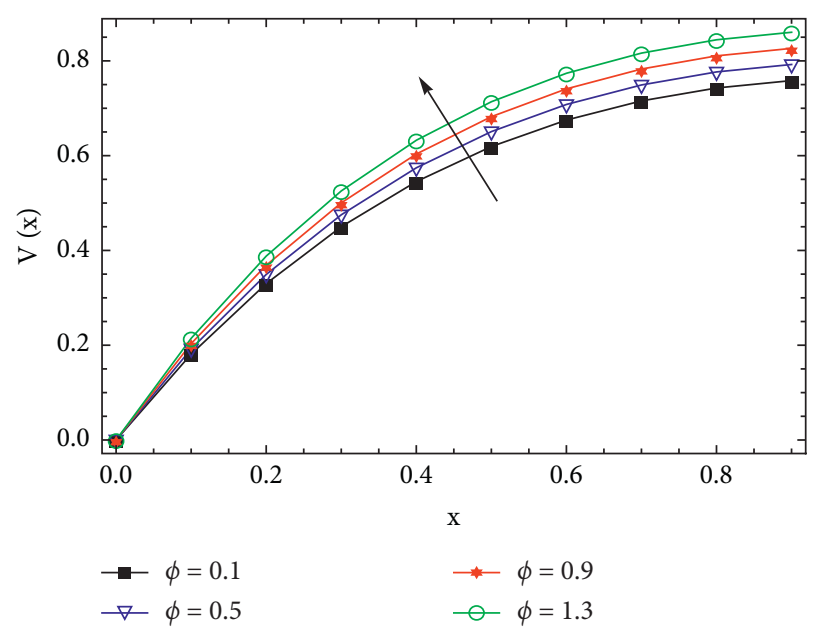

FIgure 10: The effect of $\phi$ on $V(x)$ for $\alpha=0.97, a=0.1, W_{e}=$ 1 , and $S_{t}=1$ fixed for Case 1 in the drainage case.

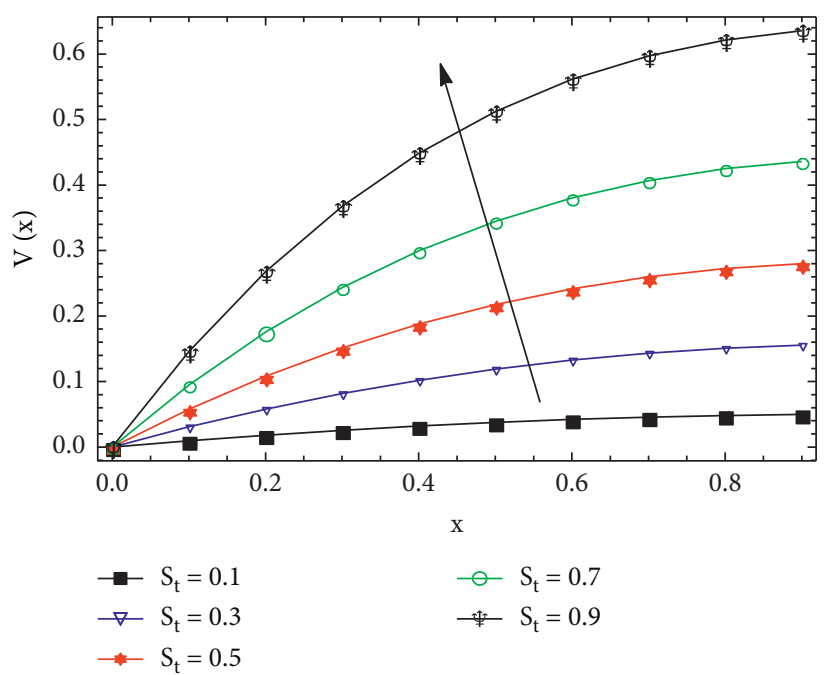

FIgURE 11: The effect of $S_{t}$ on $V(x)$ for $\alpha=0.95, a=0.2, W_{e}=$ 1 , and $\phi=0.1$ fixed for Case 1 in the drainage case. 


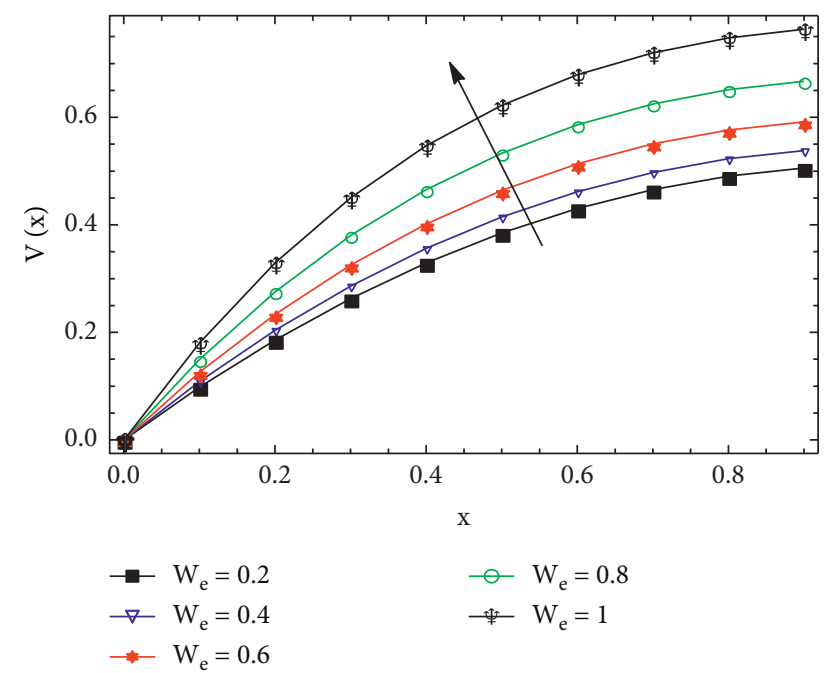

Figure 12: The effect of $W_{e}$ on $V(x)$ for $\alpha=0.95, a=0.1, S_{t}=$ 1 , and $\phi=0.1$ fixed for Case 1 in the drainage case.

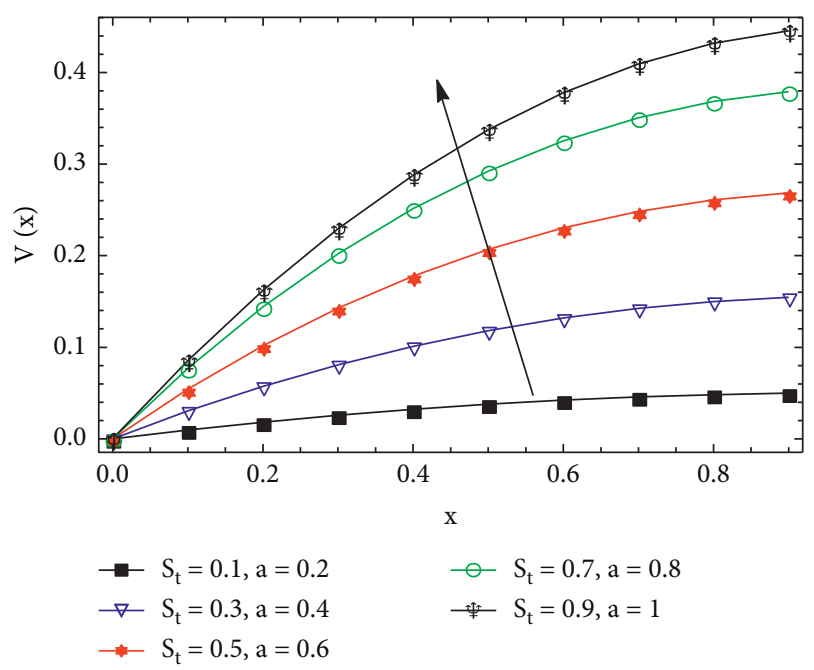

FIGURE 13: Effect of increasing $S_{t}$ and $a$ simultaneously on $V(x)$ for $\alpha=0.95, W_{e}=1$, and $\phi=0.01$ fixed for Case 1 in the drainage case.

influential parameter then $a$. Figure 7 shows the combine effect of $a$ and $\phi$. Motion into fluid particles is reduced versus higher impact of ratio of viscosity number. For higher values of $\phi$ make a declination into motion of fluid. So, fluid becomes more viscous and thick. It is seen that $V(x)$ increases with increase in $a$ and $\phi$, and hence, $a$ is more influential than $\phi$. Figures 8-14 show the effect of different parameters on $V(x)$ in Case 1 of drainage. Figure 8 depicts the effect of $\alpha$ on $V(x)$. It is seen that $V(x)$ has direct association with $\alpha$. Figure 9 shows the impact of $a$ on $V(x)$. It is seen that $V(x)$ has inverse association with $a$. Figures $10-12$ show the effect of $\phi, S_{t}$, and $W_{e}$ on $V(x)$, respectively. It is realized that $V(x)$ has direct connection with these parameters. Figure 13 shows the combined effect of increasing $S_{t}$ and $a$. It is observed that $V(x)$ increases with increase in $S_{t}$ and $a$, and hence, $S_{t}$ is more influential than $a$. Stoke number

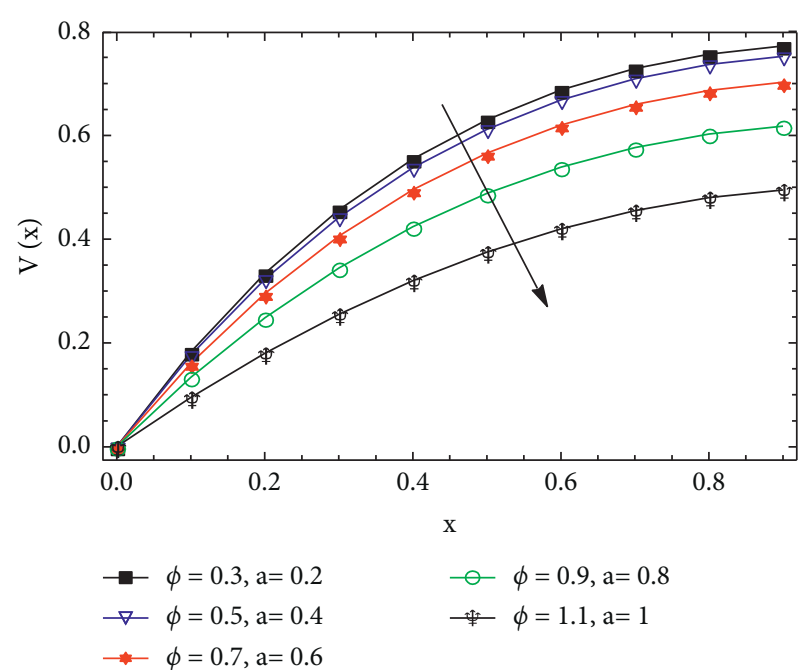

FIGURE 14: Effect of increasing $\phi$ and $a$ simultaneously on $V(x)$ for $\alpha=0.95, W_{e}=1$, and $S_{t}=1$ fixed for Case 1 in the drainage case.

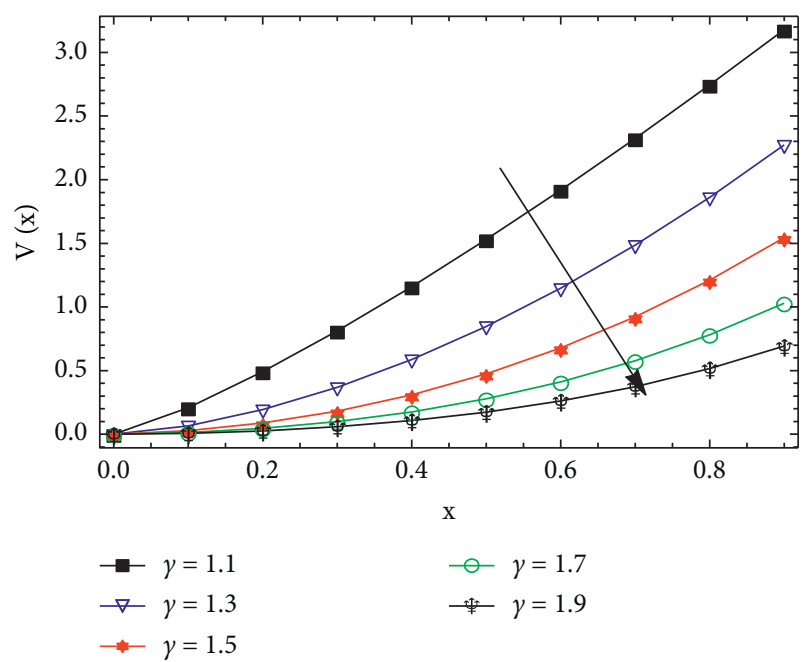

FIgURE 15: The effect of $\gamma$ on $V(x)$ for $a=0.1, W_{e}=1, S_{t}=1$, and $\phi=0.1$ fixed for Case 2 in the drainage case.

$\left(S_{t}\right)$ is a dimensionless number. Physically, it is used to know characterization of fluid particles during the flow of fluid. Basically, it is defined as the ratio among characteristics time and characteristics time during the flow regarding particle. So, it is noticed that flow of particles is directly proportional against velocity of fluid. Furthermore, Figure 14 shows the effect of increasing $\phi$ and $a$ on $V(x)$. Investigation shows that $a$ is a more influential than $\phi$. Figure 15 shows the effect of $\gamma$ on $V(x)$ in Case 2 of drainage. It is seen that $V(x)$ has inverse association with $\gamma$. Figures 16 and 17 indicate the effect of $\alpha$ and $\gamma$ on $V(x)$ in Case 3 of drainage. It is observed that $\alpha$ and $\gamma$ exhibit opposite behaviors on $V(x)$ in this case. In addition to the above results, volumetric flow and average velocities are also computed in lifting and drainage cases. 


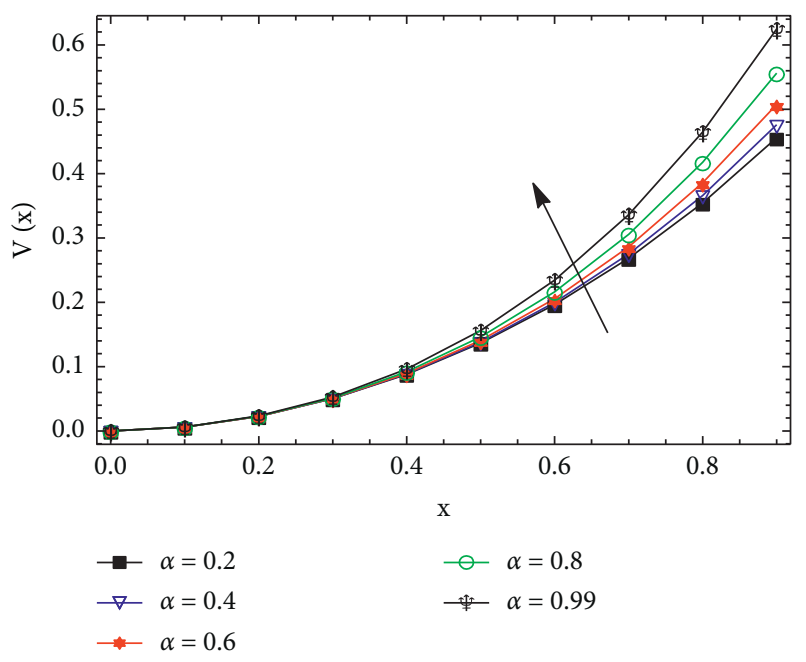

Figure 16: The effect of $\alpha$ on $V(x)$ for $\gamma=1.95, a=0.1, W_{e}=$ $1, S_{t}=1$, and $\phi=0.1$ fixed for Case 3 in the drainage case.

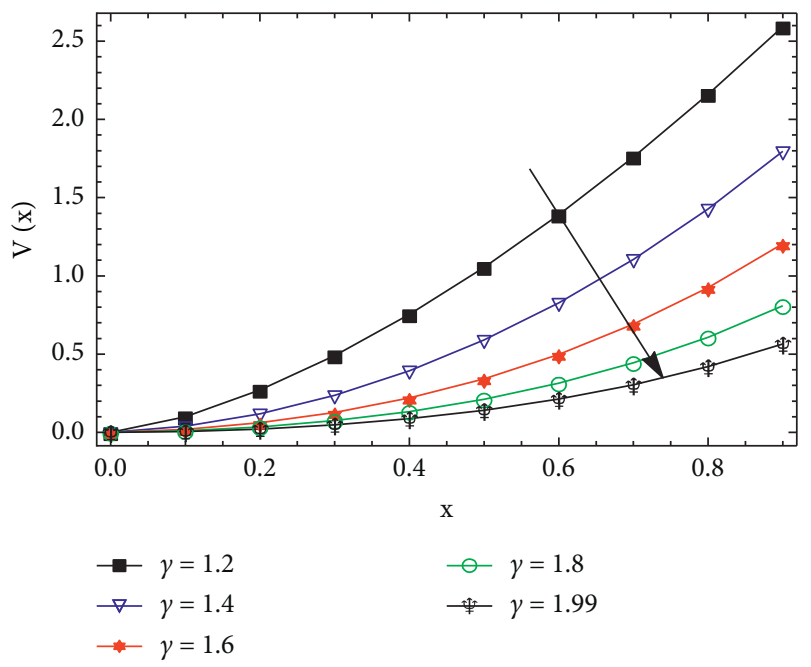

Figure 17: The effect of $\gamma$ on $V(x)$ for $\alpha=99, a=0.1, W_{e}=1, S_{t}=$ 1 , and $\phi=0.1$ fixed for Case 3 in the drainage case.

\section{Conclusions}

In this study, thin film of Johnson-Segalman fluid flow is investigated in fractional space for the case of lifting and drainage.
Solutions of boundary value problems are obtained using fractional calculus and HPM. Validity of obtained solutions is established through residual errors' computation. Few important results related to different parameters are established in the fractional space. It is comprehended that fractional parameter $\alpha$ has shown reverse effect in drainage and lifting. The study also discloses that fractional state Stokes and Weissenberg numbers showed similar effects on the fluid velocity in drainage and lifting cases. It is further seen that the Stokes number is the most influential parameter in both lifting and drainage situations. [34-39].

\section{Nomenclature}

Symbols

$\begin{array}{ll}\mathbf{v}: & \text { Vector velocity } \\ \mu: & \text { Kinematic viscosity } \\ \text { div: } & \text { Divergence } \\ \rho: & \text { Fluid density } \\ \sigma: & \text { Cauchy stress tensor } \\ p: & \text { Pressure } \\ T: & \text { Tensor } \\ \eta: & \text { Independent variable } \\ g: & \text { Gravitational force } \\ f: & \text { Body force } \\ f_{1}, f_{2}: & \text { Components of body force } \\ \alpha \gamma: & \text { Fractional parameters } \\ C_{\mu}: & \text { Real number } \\ x, y: & \text { Space coordinates } \\ T_{x x}, T_{x y}: & \text { Components of tensor } \\ \phi: & \text { Ratio of viscosities } \\ S_{t}: & \text { Stoke number } \\ \text { We: } & \text { Weissenberg number. }\end{array}$

\section{Appendix}

\section{A. Homotopy Solution in Case 1 of Drainage}

Homotopy $\Omega \times[0,1] \longrightarrow \Re$, for equation (46), is

$$
\begin{aligned}
& (1-p) \frac{d^{2} v(x)}{\mathrm{d} x^{2}}+p \frac{d^{2} v(x)}{\mathrm{d} x^{2}}+W_{e}^{4}\left(1-a^{2}\right)^{2}\left[\varphi \frac{d^{2} v(x)}{\mathrm{d} x^{2}}+S_{t}\right]\left(D^{\alpha} v(x)\right)^{4} \\
& \left.-W_{e}^{2}\left(1-a^{2}\right)\left[\frac{d^{2} v(x)}{\mathrm{d} x^{2}}+\varphi \frac{d^{2} v(x)}{\mathrm{d} x^{2}}-2 S_{t}\right]\left(D^{\alpha} v(x)\right)^{2}+S_{t}\right]=0 .
\end{aligned}
$$


Using (46) and (47), various order problems are obtained.

Zeroth-order problem:

$$
v_{0}^{\prime \prime}(x)=0, v_{0}(0)=0, v_{0}^{\prime}(1)=0 .
$$

First-order problem:

$$
\begin{aligned}
S_{t} & +2 S_{t} W_{e}^{2}\left(D^{\alpha} v_{0}(x)\right)^{2}-2 a^{2} S_{t} W_{e}^{2}\left(D^{\alpha} v_{0}(x)\right)^{2}+S_{t} W_{e}^{4}\left(D^{\alpha} v_{0}(x)\right)^{4} \\
& -2 a^{2} S_{t} W_{e}^{4}\left(D^{\alpha} v_{0}(x)\right)^{4}+a^{4} S_{t} W_{e}^{4}\left(D^{\alpha} v_{0}(x)\right)^{4}-W_{e}^{2}\left(D^{\alpha} v_{0}(x)\right)^{2} v_{0}^{\prime \prime}(x) \\
& +a^{2} W_{e}\left(D^{\alpha} v_{0}(x)\right)^{2} v_{0}^{\prime \prime}(\mathrm{x})-W_{e}^{2} \phi\left(D^{\alpha} v_{0}(x)\right)^{2} v_{0}^{\prime \prime}(x)-2 a^{2} W_{e}^{4} \phi\left(D^{\alpha} v_{0}(x)\right)^{4} v_{0}^{\prime \prime}(x) \\
& +a^{2} W_{e}^{2} \phi\left(D^{\alpha} v_{0}(x)\right)^{2} v_{0}^{\prime \prime}(x)+W_{e}^{4} \phi\left(D^{\alpha} v_{0}(x)\right)^{4} v_{0}^{\prime \prime}(x) \\
& +a^{4} W_{e}^{4} \phi\left(D^{\alpha} v_{0}(x)\right)^{4} v_{0}^{\prime \prime}(x)+v_{1}^{\prime \prime}(x)=0, v_{1}(0)=0, v_{1}^{\prime}(1)=0 .
\end{aligned}
$$

Second-order problem:

$$
\begin{aligned}
4 S_{t} & W_{e}^{2}\left(D^{\alpha} v_{0}(x)\right)\left(D^{\alpha} v_{1}(x)\right)-4 a^{2} S_{t} W_{e}^{2}\left(D^{\alpha} v_{0}(x)\right)\left(D^{\alpha} v_{1}(x)\right) \\
& +4 S_{t} W_{e}^{4}\left(D^{\alpha} v_{0}(x)\right)^{3}\left(D^{\alpha} v_{1}(x)\right)-8 a^{2} S_{t} W_{e}^{4}\left(D^{\alpha} v_{0}(x)\right)^{3}\left(D^{\alpha} v_{1}(x)\right) \\
& +4 a^{4} S_{t} W_{e}^{4}\left(D^{\alpha} v_{0}(x)\right)^{3}\left(D^{\alpha} v_{1}(x)\right)-2 W_{e}^{2}\left(D^{\alpha} v_{0}(x)\right)\left(D^{\alpha} v_{1}(x)\right) v_{0}^{\prime \prime}(x) \\
& +2 a^{2} W_{e}^{2}\left(D^{\alpha} v_{0}(x)\right)\left(D^{\alpha} v_{1}(x)\right) v_{0}^{\prime \prime}(x)-2 W_{e}^{2} \phi\left(D^{\alpha} v_{0}(x)\right)\left(D^{\alpha} v_{1}(x)\right) v_{0}^{\prime \prime}(x) \\
& +2 a^{2} W_{e}^{2} \phi\left(D^{\alpha} v_{0}(x)\right)\left(D^{\alpha} v_{1}(x)\right) v_{0}^{\prime \prime}(x)+4 W_{e}^{4} \phi\left(D^{\alpha} v_{0}(x)\right)^{3}\left(D^{\alpha} v_{1}(x)\right) v_{0}^{\prime \prime}(x) \\
& -8 a^{2} W_{e}^{4} \phi\left(D^{\alpha} v_{0}(x)\right)^{3}\left(D^{\alpha} v_{1}(x)\right) v_{0}^{\prime \prime}(x)+4 a^{4} W_{e}^{4} \phi\left(D^{\alpha} v_{0}(x)\right)^{3}\left(D^{\alpha} v_{1}(x)\right) v_{0}^{\prime \prime}(x) \\
& -W_{e}^{2}\left(D^{\alpha} v_{0}(x)\right)^{2} v_{1}^{\prime \prime}(x)+a^{2} W_{e}^{2}\left(D^{\alpha} v_{0}(\mathrm{x})\right)^{2} v_{1}^{\prime \prime}(x)-W_{e}^{2} \phi\left(D^{\alpha} v_{0}(x)\right)^{2} v_{1}^{\prime \prime}(x) \\
& +a^{2} W_{e}^{2} \phi\left(D^{\alpha} v_{0}(x)\right)^{2} v_{1}^{\prime \prime}(\mathrm{x})+W_{e}^{4} \phi\left(D^{\alpha} v_{0}(x)\right)^{4} v_{1}^{\prime \prime}(x)-2 a^{2} W_{e}^{4} \phi\left(D^{\alpha} v_{0}(x)\right)^{4} v_{1}^{\prime \prime}(x) \\
& +a^{4} W_{e}^{4} \phi\left(D^{\alpha} v_{0}(x)\right)^{4} v_{1}^{\prime \prime}(x)+v_{2}^{\prime \prime}(x)=0, v_{2}(0)=0, v_{2}^{\prime}(1)=0
\end{aligned}
$$

Similarly, we can find higher order problems and their solutions.
Third-order approximate solution after applying Caputo definition and keeping $\alpha=0.99, a=0.01, \phi=0.1, W_{e}=$ 0.1 , and $S_{t}=0.01$ fixed is

$$
\begin{aligned}
V(x)= & \frac{1}{2}\left(0.2 \mathrm{x}-0.1 x^{2}\right)+\left(0 . 0 3 8 0 9 8 2 \left(0.000092534 x^{2.96}-0.000137786 x^{4}\right.\right. \\
& \frac{\left.\left.+0.000092426 x^{5}-0.0000228777 x^{6}\right)\right)}{x^{1.96}+\left(0 . 0 7 6 1 9 6 4 \left(0.0000841218 x^{2.96}\right.\right.} \\
& \frac{\left.\left.-0.00012526 x^{4}+0.0000840236 x^{5}-0.0000207979 x^{6}\right)\right)}{x^{2.96}} .
\end{aligned}
$$

The residual is 


$$
\begin{aligned}
R= & \frac{d^{2} v(x)}{\mathrm{d} x^{2}}+W_{e}^{4}\left(1-a^{2}\right)^{2}\left[\varphi \frac{d^{2} v(x)}{\mathrm{d} x^{2}}+S_{t}\right]\left(D^{\alpha} v(x)\right)^{4} \\
& -W_{e}^{2}\left(1-a^{2}\right)\left[\frac{d^{2} v(x)}{\mathrm{d} x^{2}}+\varphi \frac{d^{2} v(x)}{\mathrm{d} x^{2}}-2 S_{t}\right]\left(D^{\alpha} v(x)\right)^{2}+S_{t}
\end{aligned}
$$

\section{B. Homotopy Solution in Case 2 of Drainage}

Homotopy $\Omega \times[0,1] \longrightarrow R$ for equation (48) is

$$
\begin{aligned}
& (1-p) \frac{d^{2} v(x)}{\mathrm{d} x^{2}}+p\left[D^{\gamma} v(x)+W_{e}^{4}\left(1-a^{2}\right)^{2}\left[\varphi D^{\gamma} v(x)+S_{t}\right]\left(\frac{\mathrm{d} v(x)}{\mathrm{d} x}\right)^{4}\right. \\
& \left.-W_{e}^{2}\left(1-a^{2}\right)\left[D^{\gamma} v(x)+D^{\gamma} v(x)-2 S_{t}\right]\left(\frac{\mathrm{d} v(x)}{\mathrm{d} x}\right)^{2}+S_{t}\right]=0 . \\
& D^{\gamma} v_{0}(x)=0, v_{0}(0)=0, v_{0}^{\prime}(1)=0 .
\end{aligned}
$$

Using (48) and (49), we obtain the following.

Zeroth-order problem:

First-order problem:

$$
\begin{aligned}
S_{t} & +D^{\gamma} v_{1}(x)+2 S_{t} W_{e}^{2}\left(v_{0}^{\prime}(x)\right)^{2}-2 a^{2} S_{t} W_{e}^{2}\left(V_{0}\right)^{\prime}[t]^{2}-W_{e}^{2}\left(D^{\gamma} v_{0}(x)\right)\left(v_{0}^{\prime}(x)\right)^{2} \\
& +a^{2} W_{e}^{2}\left(D^{\gamma} v_{0}(x)\right)\left(v_{0}^{\prime}(x)\right)^{2}-\phi W_{e}^{2}\left(D^{\gamma} v_{0}(x)\right)\left(v_{0}^{\prime}(x)\right)^{2}+a^{2} \phi W_{e}^{2}\left(D^{\gamma} v_{0}(x)\right)\left(v_{0}^{\prime}(x)\right)^{2} \\
& +S_{t} W_{e}^{4}\left(v_{0}^{\prime}(x)\right)^{4}-2 a^{2} S_{t} W_{e}^{4}\left(v_{0}^{\prime}(x)\right)^{4}+a^{4} S_{t} W_{e}^{4}\left(v_{0}^{\prime}(x)\right)^{4}+\phi W_{e}^{4}\left(D^{\gamma} v_{0}(x)\right)\left(v_{0}^{\prime}(x)\right)^{4} \\
& -2 a^{2} \phi W_{e}^{4}\left(D^{\gamma} v_{0}(x)\right)\left(v_{0}^{\prime}(x)\right)^{4}+a^{4} \phi W_{e}^{4} D^{\gamma} v_{0}(x)\left(v_{0}^{\prime}(x)\right)^{4}=0, v_{1}(0)=0, v_{1}^{\prime}(1)=0 .
\end{aligned}
$$

Second-order problem:

$$
\begin{aligned}
& D^{\gamma} v_{2}(x)-W_{e}^{2}\left(D^{\gamma} v_{1}(x)\right)\left(v_{0}^{\prime}(x)\right)^{2}+a^{2} W_{e}^{2}\left(D^{\gamma} v_{1}(x)\right)\left(v_{0}^{\prime}(x)\right)^{2} \\
& \quad-\phi W_{e}^{2}\left(D^{\gamma} v_{1}(x)\right)\left(v_{0}^{\prime}(x)\right)^{2}+a^{2} \phi W_{e}^{2}\left(D^{\gamma} v_{1}(x)\right)\left(v_{0}^{\prime}(x)\right)^{2}+\phi W_{e}^{4}\left(D^{\gamma} v_{1}(x)\right)\left(v_{0}^{\prime}(x)\right)^{4} \\
& \quad-2 a^{2} \phi W_{e}^{4}\left(D^{\gamma} v_{1}(x)\right)\left(v_{0}^{\prime}(x)\right)^{4}+a^{4} \phi W_{e}^{4}\left(D^{\gamma} v_{1}(x)\right)\left(v_{0}^{\prime}(x)\right)^{4}+4 S_{t} W_{e}^{2}\left(v_{0}^{\prime}(x)\right)\left(v_{1}^{\prime}(x)\right) \\
& \quad-4 a^{2} S_{t} W_{e}^{2}\left(v_{0}^{\prime}(x)\right)\left(v_{1}^{\prime}(x)\right)-2 W_{e}^{2}\left(D^{\gamma} v_{0}(x)\right)\left(v_{0}^{\prime}(x)\right)\left(v_{1}^{\prime}(x)\right)+2 a^{2} W_{e}^{2}\left(D^{\gamma} v_{0}(x)\right)\left(v_{0}^{\prime}(x)\right)\left(v_{1}^{\prime}(x)\right) \\
& \quad-2 \phi W_{e}^{2}\left(D^{\gamma} v_{0}(x)\right)\left(v_{0}^{\prime}(x)\right)\left(v_{1}^{\prime}(x)\right)+2 a^{2} \phi W_{e}^{2}\left(D^{\gamma} v_{0}(x)\right)\left(v_{0}^{\prime}(x)\right)\left(v_{1}^{\prime}(x)\right) \\
& \quad+4 S_{t} W_{e}^{4}\left(v_{0}^{\prime}(x)\right)^{3}\left(v_{1}^{\prime}(x)\right)-8 a^{2} S_{t} W_{e}^{4}\left(v_{0}^{\prime}(x)\right)^{3}\left(v_{1}^{\prime}(x)\right)+4 a^{4} S_{t} W_{e}^{4}\left(v_{0}^{\prime}(x)\right)^{3}\left(v_{1}^{\prime}(x)\right)+ \\
& 4 \phi W_{e}^{4}\left(D^{\gamma} v_{0}(x)\right)\left(v_{0}^{\prime}(x)\right)^{3}\left(v_{1}^{\prime}(x)\right)-8 a^{2} \phi W_{e}^{4}\left(D^{\gamma} v_{0}(x)\right)\left(v_{0}^{\prime}(x)\right)^{3}\left(v_{1}^{\prime}(x)\right) \\
& \quad+4 a^{4} \phi W_{e}^{4}\left(D^{\gamma} v_{0}(x)\right)\left(v_{0}^{\prime}(x)\right)^{3}\left(v_{1}^{\prime}(x)\right)=0, v_{2}(0)=0, v_{2}^{\prime}(1)=0 .
\end{aligned}
$$

Similarly, we can find higher order problems and their solutions. 
Keeping $\gamma=1.7, a=0.1, S_{t}=0.01, W_{e}=0.001$, and $\phi=0.1$ fixed and using Caputo definition, the approximate solution is

$$
V(x)=-0.00647381 x^{1.7}-6.77768 \times 10^{-13} x^{3.1} .
$$

The residual is

$$
\begin{aligned}
R= & D^{\gamma} V(x)+W_{e}^{4}\left(1-a^{2}\right)^{2}\left[\varphi D^{\gamma} V(x)+S_{t}\right]\left(\frac{\mathrm{d} V(x)}{\mathrm{d} x}\right)^{4} \\
& -W_{e}^{2}\left(1-a^{2}\right)\left[D^{\gamma} V(x)+D^{\gamma} V(x)-2 S_{t}\right]\left(\frac{\mathrm{d} V(x)}{\mathrm{d} x}\right)^{2}+S_{t} .
\end{aligned}
$$

\section{Homotopy Solution in Case 3 of Drainage}

Homotopy $\Omega \times[0,1] \longrightarrow R$ for equation (50) is

$$
\begin{aligned}
& (1-p) \frac{d^{2} v(x)}{\mathrm{d} x^{2}}+p\left[D^{\gamma} v(x)+W_{e}^{4}\left(1-a^{2}\right)^{2}\left[\varphi D^{\gamma} v(x)+S_{t}\right]\left(D^{\alpha} v(x)\right)^{4}\right. \\
& \left.-W_{e}^{2}\left(1-a^{2}\right)\left[D^{\gamma} v(x)+D^{\gamma} v(x)-2 S_{t}\right]\left(D^{\alpha} v(x)\right)^{2}+S_{t}\right]=0 . \\
& D^{\gamma} v_{0}(x)=0, v_{0}(0)=0, v_{0}^{\prime}(1)=0 .
\end{aligned}
$$

Using (50) and (51), we obtain the following.

Zeroth-order problem:

First-order problem:

$$
\begin{aligned}
S_{t} & +2 S_{t} W_{e}^{2}\left(D^{\alpha} v_{0}(x)\right)^{2}-2 a^{2} S_{t} W_{e}^{2}\left(D^{\alpha} v_{0}(x)\right)^{2}+S_{t} W_{e}^{4}\left(D^{\alpha} v_{0}(x)\right)^{4} \\
& -2 a^{2} S_{t} W_{e}^{4}\left(D^{\alpha} v_{0}(x)\right)^{4}+a^{4} S_{t} W_{e}^{4}\left(D^{\alpha} v_{0}(x)\right)^{4}-W_{e}^{2}\left(D^{\alpha} v_{0}(x)\right)^{2}\left(D^{\gamma} v_{0}(x)\right) \\
& +a^{2} W_{e}^{2}\left(D^{\alpha} v_{0}(x)\right)^{2}\left(D^{\gamma} v_{0}(x)\right)-\phi W_{e}^{2}\left(D^{\alpha} v_{0}(x)\right)^{2}\left(D^{\gamma} v_{0}(x)\right) \\
& +a^{2} \phi W_{e}^{2}\left(D^{\alpha} v_{0}(x)\right)^{2}\left(D^{\gamma} v_{0}(x)\right)+\phi W_{e}^{4}\left(D^{\alpha} v_{0}(x)\right)^{4}\left(D^{\gamma} v_{0}(x)\right) \\
& -2 a^{2} \phi W_{e}^{4}\left(D^{\alpha} v_{0}(x)\right)^{4}\left(D^{\gamma} v_{0}(x)\right)+a^{4} \phi W_{e}^{4}\left(D^{\alpha} v_{0}(x)\right)^{4}\left(D^{\gamma} v_{0}(x)\right) \\
& +\left(D^{\gamma} v_{1}(x)\right)=0, v_{1}(0)=0, v_{1}^{\prime}(1)=0 .
\end{aligned}
$$

Second-order problem:

$$
\begin{aligned}
4 S_{t} & W_{e}^{2}\left(D^{\alpha} v_{0}(x)\right)\left(D^{\alpha} v_{1}(x)\right)-4 a^{2} S_{t} W_{e}^{2}\left(D^{\alpha} v_{0}(x)\right)\left(D^{\alpha} v_{1}(x)\right) \\
& +4 S_{t} W_{e}^{4}\left(D^{\alpha} v_{0}(x)\right)^{3}\left(D^{\alpha} v_{1}(x)\right)-8 a^{2} S_{t} W_{e}^{4}\left(D^{\alpha} v_{0}(x)\right)^{3}\left(D^{\alpha} v_{1}(x)\right) \\
& +4 a^{4} S_{t} W_{e}^{4}\left(D^{\alpha} v_{0}(x)\right)^{3}\left(D^{\alpha} v_{1}(x)\right)-2 W_{e}^{2}\left(D^{\alpha} v_{0}(x)\right)\left(D^{\alpha} v_{1}(x)\right)\left(D^{\gamma} v_{0}(x)\right) \\
& +2 a^{2} W_{e}^{2}\left(D^{\alpha} v_{0}(x)\right)\left(D^{\alpha} v_{1}(x)\right)\left(D^{\gamma} v_{0}(x)\right)-2 \phi W_{e}^{2}\left(D^{\alpha} v_{0}(x)\right)\left(D^{\alpha} v_{1}(x)\right)\left(D^{\gamma} v_{0}(x)\right) \\
& +2 a^{2} \phi W_{e}^{2}\left(D^{\alpha} v_{0}(x)\right)\left(D^{\alpha} v_{1}(x)\right)\left(D^{\gamma} v_{0}(x)\right)+4 \phi W_{e}^{4}\left(D^{\alpha} v_{0}(x)\right)^{3}\left(D^{\alpha} v_{1}(x)\right)\left(D^{\gamma} v_{0}(x)\right) \\
& -8 a^{2} \phi W_{e}^{4}\left(D^{\alpha} v_{0}(x)\right)^{3}\left(D^{\alpha} v_{1}(x)\right)\left(D^{\gamma} v_{0}(x)\right)+4 a^{4} \phi W_{e}^{4}\left(D^{\alpha} v_{0}(x)\right)^{3}\left(D^{\alpha} v_{1}(x)\right)\left(D^{\gamma} v_{0}(x)\right)-\frac{-b \pm \sqrt{b^{2}-4 a c}}{2 a}
\end{aligned}
$$




$$
\begin{aligned}
& \cdot W_{e}^{2}\left(D^{\alpha} v_{0}(x)\right)^{2}\left(D^{\gamma} v_{1}(x)\right)+a^{2} W_{e}^{2}\left(D^{\alpha} v_{0}(x)\right)^{2}\left(D^{\gamma} v_{1}(x)\right)-\phi W_{e}^{2}\left(D^{\alpha} v_{0}(x)\right)^{2}\left(D^{\gamma} v_{1}(x)\right) \\
& +a^{2} \phi W_{e}^{2}\left(D^{\alpha} v_{0}(x)\right)^{2}\left(D^{\gamma} v_{1}(x)\right)+\phi W_{e}^{4}\left(D^{\alpha} v_{0}(x)\right)^{4}\left(D^{\gamma} v_{1}(x)\right)-2 a^{2} \phi W_{e}^{4}\left(D^{\alpha} v_{0}(x)\right)^{4}\left(D^{\gamma} v_{1}(x)\right) \\
& +a^{4} \phi W_{e}^{4}\left(D^{\alpha} v_{0}(x)\right)^{4}\left(D^{\gamma} v_{1}(x)\right)+\left(D^{\gamma} v_{2}(x)\right)=0, v_{2}(0)=0, v_{2}^{\prime}(1) .
\end{aligned}
$$

Similarly, we can find higher order problems and their solutions.

Approximate solution after applying Caputo definition and $\alpha=0.95, \phi=0.1, W_{e}=0.1, a=0.1, S_{t}=0.1$, and $\gamma=1.99$ fixed is

$$
V(x)=-0.0504625 x^{1.99}-2.39478 \times 10^{-6} x^{4.07} .
$$

The residual is

$$
\begin{aligned}
R= & D^{\gamma} V(x)+W_{e}^{4}\left(1-a^{2}\right)^{2}\left[\varphi D^{\gamma} V(x)+S_{t}\right]\left(D^{\alpha} V(x)\right)^{4} \\
& -W_{e}^{2}\left(1-a^{2}\right)\left[D^{\gamma} V(x)+D^{\gamma} V(x)-2 S_{t}\right]\left(D^{\alpha} V(x)\right)^{2}+S_{t} .
\end{aligned}
$$

Flow rate and average velocity in the drainage case is

$$
\begin{aligned}
Q= & \int_{0}^{1} v(x) \mathrm{d} x, \\
Q= & \frac{1}{6\left(15-16 \alpha+4 \alpha^{2}\right) \Gamma(4-\alpha)^{2}}\left(S _ { t } ( - 3 + 2 \alpha ) \left(\frac { 1 } { - 7 + 2 \alpha } \left(3\left(-1+a^{2}\right) S_{t}^{2} W_{e}^{2}\right.\right.\right. \\
& \left.\left.\left.\cdot(-3+\alpha)^{2}(-16+\alpha(25+\alpha(-13+2 \alpha)))(3+\phi)\right) 2(-5+2 \alpha) \Gamma(4-\alpha)^{2}\right)\right) .
\end{aligned}
$$

For drainage problem, the average velocity $\bar{V}$ is

$$
\begin{aligned}
\bar{V}= & \frac{1}{6\left(15-16 \alpha+4 \alpha^{2}\right) \Gamma(4-\alpha)^{2}}\left(S _ { t } ( - 3 + 2 \alpha ) \left(\frac { 1 } { - 7 + 2 \alpha } \left(3\left(-1+a^{2}\right) S_{t}^{2} W_{e}^{2}(-3+\alpha)^{2}\right.\right.\right. \\
& \left.\left.\cdot(-16+\alpha(25+\alpha(-13+2 \alpha)))(3+\phi))+2(-5+2 \alpha) \Gamma(4-\alpha)^{2}\right)\right) .
\end{aligned}
$$

\section{Data Availability}

All the data used to support the findings of the study are given within the article.

\section{Conflicts of Interest}

The authors declare that they have no conflicts of interest to report regarding the present study.

\section{References}

[1] C. D. Denson, "The drainage of Newtonian liquids entrained on a vertical surface," Industrial \& Engineering Chemistry Fundamentals, vol. 9, no. 3, pp. 443-448, 1970.

[2] S. B. G. O. Brien and L. W. Schwartz, "Theory and modeling of thin film flows," Encyclopedia of Surface and Colloid Science, pp. 5283-5297, 2002.

[3] H. Jeffreys, "The draining of a vertical plate," Mathematical Proceedings of the Cambridge Philosophical Society, vol. 26, no. 2, pp. 204-205, 1930.

[4] J. J. V. Rossum, "Viscous lifting and drainage of liquid," Applied Scientific Research, vol. 7, pp. 141-145, 1958.
[5] G. Astarita and G. Marrucci, Principle of Non-newtonian Fluid Mechanics, McGraw-Hill, London UK, 1974.

[6] A. M. Siddiqui, R. Mahmood, and Q. K. Ghori, "Homotopy perturbation method for thin film flow of a third grade fluid down an inclined plane," Chaos, Solitons \& Fractals, vol. 35, no. 1, pp. 140-147, 2008.

[7] A. M. Siddiqui, R. Mahmood, and Q. K. Ghori, "Some exact solutions for the thin film flow of a PTT fluid," Physics Letters A, vol. 356, no. 4-5, pp. 353-356, 2006.

[8] A. M. Siddiqui, R. Mahmood, and Q. K. Ghori, "Homotopy perturbation method for thin film flow of a fourth grade fluid down a vertical cylinder," Physics Letters A, vol. 352, no. 4-5, pp. 404-410, 2006.

[9] L. D. Landau, "On the problem of turbulence," Comptes Rendus de L'Academie des Sciences de L'URSS, vol. 44, pp. 311-314, 1944.

[10] J. T. Stuart, "On the role of Reynolds stresses in stability theory," Journal of the Aerospace Sciences, vol. 23, pp. 86-88, 1956.

[11] S. Ullah, K. Akhtar, N. A. Khan, and A. Ullah, "Analysis of thin film flow of generalized Maxwell fluid confronting withdrawal and drainage on non-isothermal cylindrical surfaces," Advances in Mechanical Engineering, vol. 11, no. 10, pp. 1-19, 2019. 
[12] Y. Raun, A. Nadim, and M. Chugunova, "Thin liquid film resulting from a distributed source on a vertical wall," arXiv: 1910.10810 [physics.flu-dyn], 2019.

[13] S. Ahmad and $\mathrm{H}$. Xu, "Mixed convection in gravity-driven thin nano-liquid film flow with homogeneous-heterogeneous reactions," Physics of Fluids, vol. 32, Article ID 023604, 2020.

[14] I. Podlubny, Fractional Differential Equations, Academic Press, New York USA, 1999.

[15] M. Yavuz, T. A. Sulaiman, F. Usta, and H. Bulut, "Analysis and numerical computations of the fractional regularized long-wave equation with damping term," Mathematical Methods in the Applied Sciences, 2020.

[16] M. Yavuz and N. Sene, "Fundamental calculus of the fractional derivative defined with Rabotnov exponential kernel and application to nonlinear dispersive wave model," Journal of Ocean Engineering and Science, vol. 6, no. 2, pp. 196-205, 2021.

[17] M. Qayyum and H. Khan, "Behavioral study of unsteady squeezing flow through porous medium," Journal of Porous Media, vol. 19, no. 1, pp. 83-94, 2016.

[18] J. H. He, "The simpler, the better: analytical methods for nonlinear oscillators and fractional oscillators," Journal of Low Frequency Noise, Vibration and Active Control, vol. 38, no. 3-4, pp. 1252-1260, 2019.

[19] X. Su, W. Xu, W. Chen, and H. Yang, "Fractional creep and relaxation models of viscoelastic materials via a non-Newtonian time-varying viscosity: physical interpretation," $\mathrm{Me}$ chanics of Materials, vol. 140, p. 103222, 2020.

[20] M. Kamran Alam, M. T. Rahim, T. Haroon, S. Islam, and A. M. Siddiqui, "Solution of steady thin film flow of JohnsonSegalman fluid on a vertical moving belt for lifting and drainage problems using Adomian Decomposition Method," Applied Mathematics and Computation, vol. 218, no. 21, pp. 10413-10428, 2012.

[21] D. J. Hashim, A. F. Jameel, T. Y. Ying, A. K. Alomari, and N. R. Anakira, "Optimal homotopy asymptotic method for solving several models of first order fuzzy fractional IVPs," Alexandria Engineering Journal, vol. 61, no. 6, pp. 4931-4943, 2022.

[22] A. Rysak and M. Gregorczyk, "Differential transform method as an effective tool for investigating fractional dynamical systems," Applied Sciences, vol. 11, no. 15, p. 6955, 2021.

[23] J. H. He, "Application of homotopy perturbation method to nonlinear wave equations," Chaos, Solitons \& Fractals, vol. 26, pp. 295-700, 2005.

[24] L. Zada, R. Nawaz, R. Nawaz, S. Ahsan, K. S. Nisar, and D. Baleanu, "New iterative approach for the solutions of fractional order inhomogeneous partial differential equations," AIMS Mathematics, vol. 6, no. 2, pp. 1348-1365, 2021.

[25] J.-H. He, "Approximate analytical solution for seepage flow with fractional derivatives in porous mediaflow with fractional derivatives in porous media," Computer Methods in Applied Mechanics and Engineering, vol. 167, no. 1-2, pp. 57-68, 1998.

[26] M. R. Yaghouti, M. Mazjini, and A. Zabihi, "Numerical solution of a family of fractional differential equations by use of RBF method," World Applied Programming, vol. 2, no. 2, pp. 88-96, 2012.

[27] Z. Liu, J. R. Clausen, R. R. Rao, and C. K. Aidun, "Nanoparticle diffusion in sheared cellular blood flow," Journal of Fluid Mechanics, vol. 871, pp. 636-667, 2019.

[28] W. Al-Kouz, C. S. Reddy, M. S Alqarni, B. Mahanthesh, and B. Mahanthesh, "Spectral quasi-linearization and irreversibility analysis of magnetized cross fluid flow through a microchannel with two different heat sources and Newton boundary conditions," The European Physical Journal Plus, vol. 136, no. 6, 2021.

[29] J.-H. He, "Homotopy perturbation technique," Computer Methods in Applied Mechanics and Engineering, vol. 178, no. 3-4, pp. 257-262, 1999.

[30] O. D. Makinde, M. T. Omojola, B. Mahanthesh et al., "Significance of buoyancy, velocity index and thickness of an upper horizontal surface of a paraboloid of revolution: the case of non-Newtonian carreau fluid," Defect and Diffusion Forum, vol. 387, pp. 550-561, 2018.

[31] S. Abbasbandy, "Homotopy perturbation method for quadratic Riccati differential equation and comparison with Adomian's decomposition method," Applied Mathematics and Computation, vol. 172, no. 1, pp. 485-490, 2006.

[32] R. Hilfer, Applications of Fractional Calculus in Physics, Academic Press, Orlando USA, 1999.

[33] J.-H. He and F.-Y. Ji, "Two-scale mathematics and fractional calculus for thermodynamics," Thermal Science, vol. 23, no. 4, pp. 2131-2133, 2019.

[34] B. R. Munson and D. F. Young, Fundamentals of Fluid Mechanics, John Wiley \& Sons, New York USA, 1994.

[35] C. S. Yih, "Stability of parallel laminar flow with a free surface," Proceedings of the Second U.S. National Congress of Applied Mechanics, pp. 623-628, ASME, New York, 1995.

[36] S. P. Py Lin, "Finite amplitude side-band stability of a viscous film," Journal of Fluid Mechanics, vol. 63, no. 3, pp. 417-429, 1974.

[37] M. W. Jr Johnson and D. Segalman, "A model for viscoelastic fluid behavior which allows non-affine deformation," Journal of Non-newtonian Fluid Mechanics, vol. 2, no. 3, pp. 255-270, 1997.

[38] A. M. Wazwaz, "Approximate solutions to boundary value problems of higher order by the modified decomposition method," Computers and Mathematics with Applications, vol. 40, no. 6-7, pp. 679-691, 2000.

[39] M. A. Noor and S. T. Mohyud-Din, "Variational homotopy perturbation method for solving higher dimensional initial boundary value problems," Mathematical Problems in Engineering, vol. 2008, pp. 1-11, 2008. 